\title{
ON THE INHERENT INCOMPLETENESS OF SCIENTIFIC THEORIES
}

\author{
Jolly Mathen * \\ San Francisco, California, USA
}

\begin{abstract}
We examine the question of whether scientific theories can be complete. For two closely related reasons, we argue that they cannot. The first reason is the inability to determine what are "valid observations", a result that is based on a self-reference Gödel/Tarski-like argument. The second reason is the existence of "meta-empirical" evidence of the inherent incompleteness of observations. These reasons, along with theoretical incompleteness, are intimately connected to the notion of belief and to theses within the philosophy of science: the Quine-Duhem (and underdetermination) theses and the observational/theoretical distinction failure. Some puzzling aspects of the philosophical theses become clearer in light of these connections. It also follows that there is no absolute measure of the information content of empirical data nor of the entropy of physical systems, and that no complete computer simulation of the natural world is possible. The connections with the mathematical theorems of Gödel and Tarski reveal the existence of other possible connections between scientific and mathematical incompleteness: computational irreducibility, complexity, infinity, arbitrariness, and self-reference. Finally, suggestions are offered of where a more rigorous (or formal) "proof" of scientific incompleteness may be found.
\end{abstract}

Key words: Theory; Observation; Completeness; Self-reference; Mathematical Incompleteness; Holism

Omnia olim mortua sunt itemur vivent.

\section{MAIN INTRODUCTION}

There is much discussion in scientific and philosophical circles on the scope and limits of science. One aspect of this discussion is whether scientific theories can ever be final or complete. For example, Weinberg (1992) and Hawking (1988, 155-169) have argued that almost all the major experimental discoveries of physics have been made and that a theory of everything (TOE) is not far off. Barrow $(1991,1998)$ and Lindley $(1993)$ have challenged this position by arguing that experimental, computational, cognitive and fiscal impediments may exist. These debates have extended beyond the domain of physics and to other disciplines in science (Horgan 1997). It has also been variously argued that parts of the world are inherently dappled (Cartwright 2001), stochastic or emergent and therefore rule out a complete scientific description based on fundamental, deterministic or reductive laws (Suppes 1978). While it is still debated whether parts of the world are actually so, it remains

*Correspondence to: Jolly Mathen, email: jkmathen@gmail.com

Received April 6, 2011; accepted May 12, 2011; Act Nerv Super (Praha) 53(1-2), 44-100.

This paper is a revision of earlier preprints that first appeared in the PhilSci and CogPrints archives in December 2004, and then with subsequent revisions in May 2005. 
possible that adequately complete descriptions may still be afforded by advances within the emerging field of complexity (Waldrop 1992; Wolfram 2002). Elsewhere, others have suggested that since Gödel (1931) proved mathematics is incomplete, any physics based in mathematics is likewise incomplete (Jaki 1966, 129; Hawking 2002). Though some have countered that certain parts of mathematics are complete and that physics may only depend on these parts (Barrow 1998, 224-227; Traub 1997a). In this regard, Wolfram (1985), Moore (1990), and Sommerer and Ott (1996) have shown that certain mathematical models of physical systems exhibit incompleteness (undecidability), but Traub (1997a, 1997b) has rejoined that it is unclear whether other mathematical or even novel physical models can circumvent these difficulties. Finally, epistemological and ontological issues within the philosophy of science, such as the observational/theoretical distinction, the Quine-Duhem (and underdetermination) theses (Duhem [1906] 1954; Quine 1951, 1960), the reality/antireality debate, and the nature of scientific explanation may impact this debate.

The above arguments can be broken down into the following categories: (a) whether the world is inherently dappled, stochastic or emergent; (b) mathematical, computational and fiscal impediments; (c) cognitive and philosophical difficulties; (d) experimental difficulties. We now ask, regardless of the issues in (a) and (b), can the debate on completeness be settled? It is the intention here to sharpen the question and not negate the importance of (a) and (b). We put forth a thesis here that ties the remaining issues, (c) and (d), together and answers the completeness question in the negative.

We begin by defining what we mean by complete scientific theories. Some may argue that theories may have to meet certain requirements of explanation, such as determinism, for the theory to be complete. On this basis Einstein, for example, famously argued that quantum theory is incomplete. Since then this requirement has been relaxed, and many physicists are willing to accept quantum theory as a foundation of a fundamental theory of physics. For our definition, therefore, we will not require a complete theory to satisfy any such "external" criteria, whether deterministic, continuous or, even, causal. We rather take our cue from the formal theories of mathematics. A scientific theory on a given domain of empirical phenomena will be said to be complete if all questions constructible within the language of the theory are answerable within the theory, or, equivalently, if for all statements constructible within the language of the theory, it is decidable whether the statement is a true or false statement of the theory. ${ }^{1}$ On this definition then, the question of determinism, etc., are all matters to be settled by the language of the theory itself.

It is immediately obvious that this definition cannot be accurately applied today due to a lack of formality in the way scientific theories are expressed. ${ }^{2}$ Therefore, we can only use it as an intuitive guide. Second, in many cases it is unclear what is the proper language of a theory and, hence, what are considered valid questions or statements. For example, in the above case of quantum theory, if we believe that particle positions are part of the language of the theory, or even of an ambient theory (world view) in which we have implicitly couched quantum theory, then the question of the whereabouts of a particle is a valid question, and the theory may be rightly considered incomplete. If, on the other hand, we exclude particle positions and instead add the notion of positional probabilities (or state vectors) to the language, then the question is not valid. Thus the question of completeness depends on what we consider to be the proper language of a theory, and if this issue cannot be settled (by scientific methodologies), then the theory is considered incomplete, a fortiori.

Even without the completeness definition and its accompanying difficulties, it is obvious today that every field of scientific inquiry stands incomplete. While some scientists believe that their fields may be approaching an end, no one will say that the goal is finished. For instance, quantum theory, regardless of the issue of determinism, stands incomplete since we do not have a quantum theory of gravity and have still many unanswered questions surrounding the behavior of subatomic particles. Practicing scientists do not need to refer to

\footnotetext{
${ }^{1}$ Though we have not given a precise definition of what is meant by a domain of empirical phenomena, the thesis to be presented here is not dependent on it, but see Section 1.3 and also Ruphy (2003, 60-62).

${ }^{2}$ Whether such a formality is ever possible is presently unclear. This possibility is discussed further in Section 3.6.1.
} 
the completeness definition to see if their theories are complete; they can simply refer to the consensus among their respective peer groups. As long as science maintains a rigorous standard, we can pragmatically claim that the definition and the consensus opinion coincide. If a time comes when the consensus deems that a respective theory is complete, then we can look to the definition for verification. If, however, a respective consensus remains negative, it is safe to say that the theory is incomplete. So while we assert in our thesis that scientific theories will remain incomplete in the sense of the completeness definition, practically we can assert that any scientific peer group will judge their respective theories to be incomplete, save for any brief periods of misjudgment.

Before giving our thesis exactly, let us elaborate on the difficulties of the cognitive, philosophical and experimental issues in regards to completeness. To begin with, the experimental dilemma concerns whether novel observations on a given domain of phenomena will cease. If they never cease, then it is uncertain whether the respective theory can ever be complete. The newly gathered empirical data may continue corroborating the theory, but there is no guarantee of this-unless the theory itself can make such a guarantee. If novel observations do cease, the answer depends on how they cease. Lindley (1993) and Barrow (1998) have pointed out that novel observations may cease due to technological and fiscal impediments, and hence could prevent the testing and determination of correct theories. This could, for example, be the case with high energy particle experiments and superstring theory. On the other hand, if upon continued experimental probing they cease (henceforth, "naturally cease"), then assuming we can get past any other difficulties, complete theories are possible. For example, Hawking $(1988,167)$ has argued that once experiments reach the Planck energy level, then no more novel discoveries are possible and we should be in a position to determine the correct fundamental theory of physics. The viewpoint that novel observations will naturally cease is essentially that natural experience is ultimately finite and therefore can be made complete, a position closely related to Russell and Wittgenstein's idea of logical atomism. In what follows, we will establish Hawking's argument more thoroughly and show how the question of theoretical completeness turns on the question of experiential completeness.

Even if empirical data is finite, philosophers of science may argue that data underdetermines theory and therefore no final theory can be singled out. Though not a direct attack against completeness, underdetermination, via its antireality implication, does lessen the epistemological force that we may normally associate with complete theories. A similar argument may be put forth on behalf of the Quine-Duhem thesis. Additionally, the inability to clearly demarcate the lines between observational and theoretical terms, and sensorial and cognitive processes within philosophy and cognitive science, respectively, exacerbates the epistemological worries. (Henceforth, both dualities will be considered to be the same. ${ }^{3}$ ) In what follows, we will argue that the situation is more grave than this; underdetermination of theory (UDT), the Quine-Duhem thesis, and the observational/theoretical distinction failure are all implicative of theoretical incompleteness. The validity of these philosophical positions, like the question on theoretical completeness, depends on the question of experiential completeness.

To answer the now larger question, we will introduce a notion that is only apparently new, but one that has been quietly stirring in the background: valid empirical observations. The main criteria that is used to judge scientific theories are observations. Thus we need to make sure that our observations are genuinely true, authentic, or valid, and that we are not mistaken or fooled by seemingly similar or even false observations. One may initially wonder what is the relevance of this notion. It usually seems obvious whether some observation has taken place (or not) and, hence, whether some theory is to be accepted (or rejected). But it is not always obvious, and as the proponents of underdetermination argue, there exist cases for

\footnotetext{
${ }^{3}$ Indeed, both Jerry Fodor (1984, 1988) and Paul Churchland (1979, 1988) have used results from cognitive science to respectively argue for and against the observational/theoretical distinction; however, the still developing field of cognitive science may prove that an identity between the philosophical and cognitive dualities is inaccurate. Nonetheless, a stance for or against either duality will have some impact on the other.
} 
which interpretational issues preclude a clear determination of observations. Even in the cases of a clear determination, it would be reasonable to ask for some procedure, preferably scientific, that could assure us whether the said observations have taken place or not, so that we may dispel any remaining doubts and safely ignore the lone skeptic in the corner.

Now if one is a fallibilist, she does not, of course, need nor expect an assurance of observational validity. In this regard it is reasonable to claim that our observations (and theories) are fallible at least to some extent and, hence, no such assurance can ever be total. One reason for this is that empirical practice (and science broadly) cannot be reduced to pure logical deduction (in the strict mathematical sense); it has many non-logical, yet reasonable aspects. Other reasons may include some of the hurdles facing complete theories, as was mentioned at the start of this introduction: a dappled or stochastic world, computational difficulties, etc. It is not our purpose here to address these issues, but to inquire whether observations can be valid (or fallible) in another, perhaps more fundamental sense-one which may, however, at least partially, underlie some of these former issues. Particularly, we are interested in how thorough our observational procedures can be in light of the aforementioned experimental, cognitive and philosophical difficulties. If one is a fallibilist because she already believes that some of these aforementioned (or related) difficulties are indeed problematic-for example, she believes that certain philosophical difficulties with empirical models of confirmation point to a holistic interpretation of science-then she also does not need nor expect any assurance of observational validity. Though moreover, in such cases, the arguments being presented here may then be related and supportive, depending on her specific position and the degree to which she holds it.

In what follows, we will argue that the acceptance of theoretical completeness and the observational/theoretical distinction, and the rejection of UDT and the Quine-Duhem theses depend not only on experiential completeness, but also on the existence of valid observations, two closely related notions. Finally, to argue our thesis that any scientific theory is inherently incomplete, we will show that there can exist no scientific procedure to determine valid observations and, second, that there is "meta-empirical" evidence to support the thesis of experiential incompleteness.

The demonstration of the non-existence of a procedure to determine valid observations is based on a self-reference Gödelesque argument. However, it is important to note that this demonstration is not based on the traditional argument stating that any math based physics (or science in general) is incomplete, owing to Gödel's proof (1931) that math is incomplete. It is a more fundamental argument that we apply to all scientific theories regardless of whether their mathematical models exhibit undecidability or whether they even have mathematical models. ${ }^{4}$ But in another sense, while we are not concerned with the traditional arguments of mathematical and computational difficulties, by examining Gödel's results in depth, we can gain a deeper understanding of scientific incompleteness.

The theorem of undefinability of valid observations (as it will be referred to henceforth) is actually more parallel to Tarski's undefinability theorem (1933) than to Gödel's incompleteness theorem. Shortly after Gödel, Tarski used Gödel's methods of self-reference to show that the notion of arithmetical truth is formally undefinable within arithmetic (i.e., that all true arithmetical statements are not recursively enumerable). ${ }^{5}$ While this implies that arithmetic is not finitely axiomatizable, it comes short of actually producing an undecidable arithmetical statement, Gödel's accomplishment. Similarly, we do not produce an actual undecidable scientific statement, but only show that the notion of valid observations, the

\footnotetext{
${ }^{4}$ There is a caveat concerning this statement, namely, that all scientific theories may ultimately turn out to be mathematical in character; moreover, they may all be mathematically rich enough (as the arithmetic of natural numbers) to manifest incompleteness. In this regard, the demonstration of undecidability in the mathematical models of certain physical systems, as mentioned in the first paragraph, may be relevant. However, the current difficulty in establishing undecidability in every mathematical manifestation of a theory and for every theory leaves us to consider the present line of argument. The prior possibility is further discussed in Section 3.6, where we consider possibilities for more rigorously establishing scientific incompleteness.

${ }^{5}$ It is now recognized that Gödel independently made the same discovery while working on his incompleteness theorems (see, for example, Murawski 1998).
} 
primary criteria to determine scientifically true theories, is undefinable within scientific theories. Moreover, we will argue that, like the implication from Tarski's theorem, our theorem implies that scientific theories are also not "finitely axiomatizable" and are therefore incomplete. The current inability to produce the scientific counterpart to Gödel's theorem and the lack of more formality in the demonstration of our theorem is due to the lack of a formal metatheory of scientific explanation. The possibility of such a metatheory and other routes for more rigorously establishing scientific incompleteness will be discussed.

We also note here that Breuer's theorem (1995), stating that no observer can distinguish all states of a system in which he is contained, which was also proved using a Gödelesque selfreference argument, maybe related to our theorem, although this is not presently clear. What is clear, as Breuer points out, is that the language of scientific theories is capable of selfreference since it contains concepts and expressions referring to its own propositions: "If apparatus and object system, as well as their interaction, can be described by the theory, then the semantic concept of observation can be introduced into the language of the theory" (201). For our purposes, we will also add the sensorial-cognitive system of the human being to what can be described by the theory. Because it is this system that determines whether observations are valid or not, we can introduce the enlarged concept of valid observations into the language of the theory.

Closely related to the notion of valid observations is that of complete observations. We will argue that they are mutually implicative. Therefore, evidence of observational incompleteness would, in addition to directly supporting the thesis of theoretical incompleteness, also remain consistent with the theorem of undefinability of valid observations. We contend that observational incompleteness is not purely an external characteristic of nature, but inherent to our experience of nature. It is a consequence of our sensorial-cognitive interactions with nature. We will argue that there is meta-empirical evidence to support the thesis of observational incompleteness. The primary evidence is the precision gap that accompanies any physical measurement. Experimental measurements are never $100 \%$ precise; there is always room for improvement. It is in this room where novel experiences lie. We will also identify three other components of an observation that can contribute to novel experiences: perspective, interaction and range.

We point out here that novel experiences do not necessarily mean anomalous experiences. They simply mean previously unexperienced, for example, the discovery of a new particle or a more precise measurement of some physical property. These novel discoveries may still continue corroborating the current theories, or they may not (an anomaly). If they forever do, then novel experiences become a moot point. Presently no scientific theory, or meta-scientific theory, can guarantee that more precise measurements will continue corroborating a theory. Unless such a guarantee is given, it will remain uncertain whether improved measurement precisions will result in anomalies or not. Because of the theorem of undefinability of valid observations and other surrounding arguments given herein, we will assume that such a guarantee cannot be given and, hence, that novel experiences pose a relevant uncertainty for the completion of scientific theories. However, this assumption is not justified by the metaempirical evidence alone. Nonetheless, future research may reveal empirical evidence that further justifies this assumption, and perhaps even show that continued novel experiences will necessarily result in anomalies.

Experiential incompleteness, or the novel experience problem, as it will be otherwise called, while also supporting UDT and the Quine-Duhem theses, clarifies some of their puzzling aspects. For example, how is it by UDT that there can exist empirically equivalent but theoretically distinct theories? We will argue that it is only so because the empirical data itself is somewhat fuzzy or, otherwise, incomplete; if the data set on an empirical domain is completely known, then only one isomorphic class of theories can exist on that domain.

Finally, we will argue that scientific incompleteness, via UDT and the Quine-Duhem theses, is intimately connected with the notion of belief, whatever form it takes, religious or otherwise. This is a consequence of an inherent incompleteness. Belief and understanding as cognitive capacities are related by the incompleteness of the latter. Faith and doubt are only 
possible because of the inherent incompleteness of our ideas about the world, or vice versa: theories cannot be complete because of our fundamental cognitive capacity to doubt them and believe in some other theory. Already we can see that this is connected to the theorem of undefinability of valid observations: e.g., how do I know that this is truly a glass of water sitting before me? We can always ask what may lie behind our concepts and experiences. It will be argued that this is not such a trivial inquiry as, for example, Horgan (1996, 29-31) suggests in his discussion of ironic science and Francis Bacon's plus ultra ("more beyond"). What is fact and what is belief cannot be so easily separated.

It is on this point that we will begin the paper. We start in Part I by comparing the philosophical debate on God's existence to the debate on whether a physical theory of everything is possible. By this comparison, we can gain an intuitive understanding of the connection between incompleteness and belief, and also of some of the other key ideas mentioned thus far. The theorem of undefinability of valid observations will be introduced at the end of this part. In Part II we will discuss in detail the relations between scientific incompleteness and the above mentioned philosophical theses, and give a more thorough exposition of the novel experience problem. At the end of Part II we will present an informal quantification of our philosophy. Theorems will be introduced that quantify the relations between the Quine-Duhem (and UDT) theses and theoretical and experiential incompleteness. Additionally, it will be shown that the novel experience problem implies there is no absolute measure of the information content of empirical data nor of the entropy of physical systems, and also that no complete computer simulation of the natural world is possible. Lastly in Part III, we will take up a thorough examination of mathematical incompleteness and establish the conditions under which incompleteness manifests, such as computational irreducibility, complexity, and infinity, and argue how these conditions could also exist in science. We will also argue how mathematical self-reference and arbitrariness find scientific parallels in the observational/theoretical distinction failure and the QuineDuhem (and UDT) theses, respectively. At the end of Part III we will offer some suggestions for where we might find an incompleteness theorem for science. For clarity, the structure of the paper charts an ascending road, from basic intuitive notions to robust philosophical ideas and, finally, to formal mathematical concepts.

\section{PART I: TOES AND THE UNDECIDABILITY OF GOD}

1.1. Introduction. After centuries of debate, it is widely acknowledged in philosophical circles that the question of whether a God exists is ultimately undecidable. (By undecidable we mean that it is neither provable that God exists nor provable that he does not.) Also presently, there is some debate within the scientific and wider communities whether a theory of everything in physics (TOE) is possible. We will show that these two debates are not unrelated but share a common thread. A resolution to either one requires the mutual consideration of both. We begin therefore by examining the God existence debate within the empirical arena of science.

But first let us briefly clarify our definition of God, as there are many. We are concerned with the definition (or part of the definition) of God as a supernatural, omnipotent being, a being who is able to defy the laws of the natural world, for example, the laws of physics. This definition can be associated with the Gods of western religions. We are not concerned here with definitions of God that, instead of assigning supernatural powers, may portray him, her or it as a passive observer, an all pervasive consciousness, a living but empty presence, etc., as can be found in many eastern religions. Although the latter definitions may have some peripheral bearing on this paper as a whole, it is not relevant to the central argument in this part.

1.2. The God Existence Debate. A decision is unreachable on two opposing counts: the inability to prove God does exist and the inability to prove he does not. Let us examine both 
counts briefly. First, why can we not prove God does exist? We consider three cases. Say we witness an unlikely, though not physically impossible event, such as a mist in the shape of a cross appearing on a window, water running from the eyes of a holy statue or the spontaneous organization of gas molecules into the corner of a room. The theist will argue that such occurrences are highly unlikely to the point of impossibility and so can only be caused by supernatural causes. But the atheist will counter and say that even highly unlikely events are not completely impossible and so does not necessitate belief in a God.

Next, consider the case where it appears that a physical law is broken (a miracle). Examples may include an object falling upwards in violation of the law of gravity or a particle annihilation event that violates the laws of particle physics. The theist will argue that only God, who is omnipotent, could break the laws of physics and cause such events to occur. But the atheist would counter that the laws of physics were never complete and therefore leaves room for surprises and new theories. She may cite as examples the startling predictions of the heliocentric, relativity or quantum theories. Once again, assent is not required.

Finally, consider the case where we witness the appearance of God, Herself. This may be a physical manifestation of any form, which also appears to be performing acts of physical impossibility. The theist will simply say, "What more proof do you need?" But alas, the atheist can counter with the possibility that some advanced alien civilization, whose knowledge of physical laws is far superior to ours, is orchestrating a complex technological drama for their curiosity or amusement. This counter argument goes through for the same reasons as the previous case: the knowledge of physical laws thus far is incomplete.

Of all three cases, the last two are the most interesting. This is because the breaking of physical laws would require assent, whereas the occurrence of an unlikely event would only suggest assent, albeit strongly.

Now let us examine the other side of the coin: why we cannot prove that God does not exist? After considering the three cases before, we only need to consider one line of argument here. Unfortunately, for the atheist, the failure is due to the same reason she used in the previous two cases to argue against the existence of God: the knowledge of physical laws thus far is incomplete. For the theist will argue that since physical laws are incomplete, there are physical observations that the laws cannot yet explain, and thus how do we know that God is not responsible for them? Of course the atheist will argue that prior observational anomalies have been resolved by new physical theories without the need for supernatural explanations. But then the theist will rejoin that the resolutions always left new anomalies unexplained.

Moreover, the theist will argue that since the whole of physical theory is not complete, how do we in fact know that the theory is correct? How do we know that God is indeed not responsible for the whole of observational phenomena, not just the unexplainable ones? To wit, how do we know that God is not the total and complete theory of everything? And finally, therefore, how do we know that God did not in fact cause the above mentioned unlikely or physically impossible events?

Let us now succinctly summarize both sides of the debate. Any empirical evidence (or miracles) of God are unconvincing because physical theories are thus far incomplete, and therefore loopholes allow for some yet unforeseen new theory to alternatively account for the said empirical evidence. On the flip side, the lack of a complete physical theory allows for God to slip in and account for the said empirical evidence. Precisely put: in lack of a complete fundamental physical theory, such as a TOE, empirical evidence cannot be said with certainty to fall within the purview of science or of God. The question of God's existence is empirically undecidable because of the incompleteness of physical theories.

1.2.1. Analysis of the Debate. At this point we are making no claim concerning the existence or non-existence of God. For instance, some may argue that empirical evidence is converging toward a complete theory and that the God of the gaps is being squeezed out, once and for all. Others may maintain that there will always be new and unexplainable 
natural phenomena. Neither of these viewpoints are being disputed as of yet. We are only making a logical point: as long as we do not have a complete fundamental theory that accounts for all natural phenomena, and there remains some gap, no matter how small, we must admit that the question of God's existence is undecidable.

With this in mind, we now ask two questions that does force us to take sides: is it possible to close the gap and achieve a complete physical theory and thus settle a debate which has been plaguing philosophy for centuries? Second, and more preliminary, does the question of God's existence, a religious question, and the question of achieving a TOE, a scientific one, really have any bearing on one another? In other words, cannot one maintain belief or disbelief in God irrelevant of physical theories? In what follows we will see that these two questions are only apparently different.

Let us begin our analysis with the second and preliminary question. Can we still have faith in God in spite of a TOE? Does a TOE necessarily exclude God from having any explanatory role in our universe? For the theist may argue: so what if we have a physical theory that can explain all observable physical phenomena, how do we know that God is not still behind it all? How do we know that God is not responsible for seeding the big bang? How do we know that God is not ultimately the source behind the fundamental particles or entities, the quarks, superstrings or whatever we find them to be eventually? How do we know that God is not behind our senses and thoughts, behind our conscious awareness itself? By raising these questions, the theist is implying that there is room for religion beyond science, that science is not the whole story, and that in the end science cannot cripple his belief in a deity or any other notion.

In order to answer our question of whether science has any bearing on issues of religious belief, we need to ask ourselves whether these questions raised by the theist are truly beyond the reach of science and a TOE, or is it instead that we have jumped prematurely in crowning a purported TOE, where in fact we have yet to produce a more thorough and fully complete theory that leaves no questions unanswered for the theist? That is to say, once we have a TOE, should there be any fundamental questions left at all? In order to answer this, we need to have a clearer understanding of what it is that we expect from a TOE. A clearer understanding of these expectations are also necessary before we can say whether we can achieve a TOE or not, and thus answer the first of our questions above.

1.3. Definition of a TOE. Before we start with our definition of a TOE, let us briefly address two preliminary issues. The first issue is the scientific scope of a TOE. Is it limited to fundamental physics, all of physics, or all of the sciences? Surprisingly (and as we will see in Part II), for our arguments it does not matter. Furthermore, it does not matter whether we are talking about a TOE or some other scientific theory which only addresses a limited (local) domain of phenomenological experience. The issue is whether the theory is complete on its given domain of phenomenological experience. It is easy to see that the above arguments concerning TOEs and God hold for these lesser grandiose theories. What complicates the issue is whether domains of phenomenological experience (physics, chemistry, biology, etc., and specialties within these fields) can be clearly distinguished. If they cannot, then theories, in order to be considered complete, may have to explain more than what was initially expected of them, or may require the support of auxiliary or background theories. For example, if a theory of chemistry is to be considered complete, it may have to explain the behavior of subatomic particles and also complex organic molecules, such as DNA and proteins. Though reductionism - vertical and horizontal-has been invoked to answer this call, scientists and philosophers continue to debate its merits.

While we admit these questions to be open but impertinent to the core of our arguments, we, nonetheless, take the viewpoint that all phenomenological experience is connected and that reductionism relative to a fundamental theory of physics holds. The former allows us to address the entire domain of phenomenological experience without worrying whether it can be precisely categorized; the later allows us to delineate our TOE requirements in a concise manner-since we limit ourselves to the domain of physics alone-and thus streamline our 
discussion. In what follows, one is free to just as well assume that our TOE concerns only the domain of fundamental physics and also that it is not a reduction of other scientific theories. The relevant question we are concerned with is whether the theory can be complete.

The second issue is whether mathematical and computational difficulties will prevent us from answering certain questions in spite of having achieved a TOE. An example of one such category of problems is what is know as intractable, a specific possible instance being that of how amino acid sequences fold into proteins (Traub 1996, 1997b). It is possible that some or all of these mathematical and computational difficulties can be overcome with new theoretical or technological breakthroughs (e.g., quantum computing). For our discussion we will assume hypothetically that all such technical difficulties can be overcome. We then ask are there still relevant questions remaining? For we are interested in whether a TOE, regardless of mathematical and computational difficulties, can answer all our questions (but see footnote four).

1.3.1. Unification. We now begin our definition of a TOE with some requirements that most physicists would agree on. It should unify all the four known fundamental forces or interactions: gravity, electromagnetism, strong and weak. ${ }^{6}$ In this unification there should be a reconciliation between the two main but disparate theories of modern day physics, Einstein's theory of gravity and quantum mechanics. This reconciliation should address large mass/energy phenomena, such as that which is theorized to occur in the singularities and vicinities of black holes, the earliest parts of the big bang, and in particle physics extending to the Planck energy scale. The TOE should also answer all of the questions concerning the strong nuclear interaction which our current theory leaves unanswered. It should resolve the missing mass cosmological anomaly (Zwicky 1933; Babock 1939) and the recently observed acceleration of the expansion of the universe (Perlmutter 1988; Zehavi 1999). Additionally, any new interactions or processes discovered as a result of future research (e.g., during empirical verification of the TOE) should also be explained.

1.3.2. Uniqueness. Next, we continue with some TOE requirements that enjoy a less certain, but still prominent status among physicists. These comprise an explanation of the parameters and classifications of the Standard Model of particle physics, including the origin and masses of the elementary particles, along with their charge, spin, color and other quantum numbers; the relative field strengths of the four interactions; the preference for matter over antimatter; the various symmetries and symmetry violations; the values of the physical constants, such as the cosmological constant; the number of dimensions of our universe; finally, and if possible, how exactly the universe began and will end (what physicists term the boundary conditions-at present our theories cannot account for the very beginnings of the big bang). Why the uncertainty over these criteria? For a long time it was assumed, or at least hoped, that a future TOE would provide some or all of these details as a by-product of unification, but the progression of theoretical research towards a TOE suggest that this may not necessarily be possible.

As an illustration of these reservations, let us consider the currently popular theoretical candidate for a TOE, string theory. This eleven-dimensional theory does a beautiful job of unifying the different fundamental interactions and the many elementary particles as the distinct features of a topological landscape of higher dimensions rolled up too tiny for us to see; however, there are tens and thousands of ways these higher dimensions can be rolled up, or compactified, and only one of these will yield the specific details of the interactions and particles that we observe in our universe, i.e., the physical properties of our universe. The problem is that the theory does not pick out a unique compactification; there are many compactifications that will yield a consistent theory, albeit describing a universe with a different set of physical properties. Theorists are uncertain whether string theory, or some

\footnotetext{
${ }^{6}$ The electromagnetic and weak interactions were unified circa 1970 in the electroweak theory.
} 
improved version of it (e.g., $\mathrm{M}$ theory), should necessarily predict the values unique to our specific universe.

Some believe that it should and are arduously pursuing this cause (Greene 2003, 218, 360361); some have even proposed plans for a solution. For instance, Hawking (2003) and others have argued that the physical properties are not uniquely determined just as the position of a subatomic particle is not; the different solutions are represented as quantum mechanical amplitudes with various probability outcomes. The idea is that many different types of universes, each with a different set of interactions and particles could have evolved at the earliest stages of the big bang, and it just so happens that ours was the one that did. Smolin (1997) has suggested that the physical properties have evolved via natural selection as universes continually give birth to new universes during black hole formations. Similarly, Linde (1994) has proposed that universes are continually sprouting new and different universes via the Guth inflationary process that is believed to have occurred during the big bang.

Others are not convinced that the uniqueness question can be answered and are simply hoping to find a match between one of the possible compactifications and the properties observed in our universe-a daunting mathematical task in itself (Greene 2003, 219-221). This group is flexible in admitting the anthropic principle (Susskind 2003) or religious explanations (Davies 1992, 161-193) as the determining reason. They are willing to accept the possibility that these details are simply beyond the reach of science, leaving an opening for the theist to enter his wedge of questions. Of course, the former group maintains that there is no opportunity for the theist here; the TOE is still incomplete. Who is right?

1.3.3. The Entities of String Theory. Let us for the moment assume that the uniqueness question can be answered satisfactorily. Then consider what we have thus far accomplished: a unification of the four fundamental forces, an elegant explanation of the many messy details of the Standard Model, an explanation of why we macroscopically observe three space and one time dimension. While we are at it, let us also assume that somewhere in these explanations reside accounts of the missing mass anomaly, the acceleration of the universe, black hole singularities, and how exactly the universe began and will end. Is that it? Have we succeeded in completing a TOE?

Perhaps some will feel that the theory should tell us what these eleven dimensional "strings" really are? What is the nature of their substance? Energy? What form of energy? Or is it holographic and informational in nature as some have suggested (see Greene 2003, 411 for references)? If so, how does information as a foundation give rise to material particles? Some might ask why specifically eleven dimensions (or 26, or however many we eventually determine there to be)? Why should entities exist in any sort of dimensional space in the first place? What in fact are dimensions? Are the group of physicists who were earlier holding out for an explanation of the uniqueness of our universe now satisfied and willing to dismiss these new questions as truly beyond science?

1.3.4. Quantum Mechanics. Elsewhere, others might still question, even after almost a century of service, whether quantum mechanics is a complete theory and the appropriate foundation upon which to build a TOE. Although it has proven to be a highly accurate theory, there are many mysteries surrounding it: (a) What actually happens during a superposition of quantum states? (b) Can the measurement (wave function collapse) problem be solved? (c) How are non-local correlations to be understood? Today it is unclear whether these mysteries are indispensable features of the theory or whether they can be resolved by an alternative interpretation, or even by some new theory. Though Bohr $(1949)$, Feynman $(1967,129)$ and other physicists have felt that these mysteries are either irrelevant or have been already answered by quantum theory and its standard interpretation (the Copenhagen 
interpretation $)^{7}$, a relatively recent poll has found that many physicists today feel that these mysteries are relevant and open (Durrani 1999).

In this spirit, there have been numerous attempts to fill the gaps. These include the novel reinterpretations of quantum theory such as the many worlds (Everett 1957; de Witt 1970), consciousness collapse (Wigner 1961), quantum event (Hughes 1989), relational (Rovelli 1996), correlation/Ithaca (Mermin 1998) and information theoretic (e.g., Zeilinger 1999). Reinterpretations that seek to re-establish the classical deterministic view over the probabilistic one ushered in by quantum mechanics include the various hidden variable theories (Bohm 1952; Dieks 1991), among others. None of these reinterpretations have received general acceptance from the physics community; they are variously debated, considered incomplete, and continue to be articulated and developed by their respective adherents. There also remains the possibility that these reinterpretations may actually be new theories that can be experimentally distinguished from the original quantum theory. The acerbity of this issue is obvious in attempting to interpret Hawking's quantum mechanical explanation for the uniqueness of our universe (Section 1.3.2). For example, if we subscribe to the many worlds interpretation, then we hold that thousands of different universes were created with the big bang and we live in just one of them. Is this in fact the case, or perhaps some other interpretation, or even some new theoretical explanation besides quantum mechanics? Or do we leave well enough alone and crown our TOE?

Others have concentrated their efforts on the measurement problem and have put forth theoretical addendums to quantum theory that provide novel experimental predictions. These include a modification of the hidden variable theory by Bohm and Bub (1966), the dynamic collapse theories (Ghirardi 1986; Gisin 1992), some still developing quantum gravity theories (Smolin 2003 for a summary), and the traditional decoherence theory. Though the hidden variable, dynamic collapse, and quantum gravity theories have yet to be experimentally corroborated, the decoherence theory has recently received some corroboration from the remarkable SQUID experiments, which possibly demonstrate the existence of quantum superposition states for macroscopic size objects (Friedman 2000; van der Wal 2000). Despite this preliminary success, the theory's lack of a direct causal mechanism for wavefunction collapse leaves some to wonder whether it can provide a final resolution to the measurement problem (Adler 2003; Zeh 2003, 14-15).

Last, we mention some significant theoretical and experimental developments that cause great difficulties for a resolution to these mysteries in terms of our traditional, intuitive concept of properties (that belong to objects or physical systems). Gleason (1957) has implied, and Kochen and Specker have shown (1967) that from purely mathematical arguments that the probability distributions that quantum mechanics predicts cannot simply be statistical distributions about properties that are themselves fundamentally non-statistical. This means that if the statistical predictions of quantum theory are as experimentally accurate as they seem to be, then electrons, for example, do not inhere with any definite momentum value. However, the fact that a value is produced upon measurement prompts many to insist that the electron has at least some contextual momentum value that depends on the measurement procedure. Additionally, Bell (1964) has shown that any physical theory that is noncontextual concerning properties (i.e., objective) and also local (i.e., does not allow faster than light connections between spatially separated particles) will produce statistics that are different than what quantum theory predicts for certain prescribed experiments. These experiments have been carried out by Aspect $(1981,1982)$ and Rowe (2001), among others, and thus far support the statistics of quantum theory. Many take these results to imply that any interpretation, addendum or replacement of quantum theory will have to be non-local and treat physical properties as contextual at best, or disregard them altogether at worst; however, some still see possibilities for a traditional recovery (Barut 1995; Bub 1995).

The former viewpoint poses severe ontological difficulties for the future of quantum theory. First, some reconciliation is in order with the special theory of relativity, for although

\footnotetext{
${ }^{7}$ Many may instead take von Neumann's operational interpretation ([1932] 1955) as the standard—perhaps more orthodox-or even conflate it with the Copenhagen.
} 
it has been shown that the non-locality of quantum mechanics does not allow information to be transmitted faster than light, there is a non-local influence of a subtle kind. Second, and more gravely, if particles and systems do not have objective properties, what do they have? Perhaps it is wise not to ask any more questions, as Bohr and Feynman felt, and simply get on with the application of the theory. Perhaps such questions are beyond the domain of science and we should be content with quantum theory as it is. Or perhaps they point towards a new physical theory in which long trusted concepts such as object, property, space and causation must be reconsidered. ${ }^{8}$ Or even, perhaps, there may yet be a resolution couched in the traditional concepts.

1.3.5. Before the Big Bang. Another area where we might question whether our TOE has fulfilled all its requirements is in the explanation of the universe's origin. Even if we could understand the big bang all the way back to its very start, can we not still ask what came before the big bang? It may be true that time in our universe started with the big bang. But is there a larger arena where the life and death of our universe takes place? Possibilities are the "multiverses" of Smolin and Linde, where individual universes created via black hole formation and inflationary expansion, respectively, are sprawled over vast stretches of space and time. So it may be a valid scientific question to ask what came before the big bang.

1.4. Resolution: Incompleteness. Although there may be further requirements for a TOE that we have omitted, we can already see from our discussion thus far that there is some uncertainty over the requirements considered here. It may perhaps help us to list some of these requirements in order of consensus among the physics community, with the first being generally agreed upon and the last being highly questionable: (1) unification of the four forces, and unification of quantum mechanics with general relativity, (2) an account of the uniqueness of the physical properties of our universe, (3) an explanation of the entities of string/M theory, (4) a better understanding of quantum mechanics and subatomic phenomena, (5) an account of what came before the big bang. This list is only based on what we know now and assumes that, in our attempts to meet any of these requirements, we will not encounter additional fundamental questions whose status as a TOE requirement may also be debatable.

We now note that the above list loosely reflects some of the theist questions in Section 1.2.1. Five can be compared with, "How do we know that God did not create the big bang?" Two and three can be compared with "How do we know that God is not ultimately the source behind the fundamental particles?" We once again ask are these questions the domain of science or religion? What ontological criteria can we use to make such a distinction? How is the question, "What is an atom made of?" distinguished religiously or scientifically from the question, "What is a string made of?"; or "What gives rise to mass?" from "What gives rise to dimensions?"; or "How was the universe created?" from "How was the big bang created?"? Without invoking something arbitrary, it appears that there can be no ontological criteria to distinguish the two domains. This immediately implies that these questions can fall on either side of the line. Such an implication however goes counter to our intuition of scientific facts on the one hand, and religious belief on the other.

A resolution to this conflict can be found along the following lines. For any given question about our phenomenological experience, we can pursue a scientific course of inquiry; however, this effort cannot yield a complete answer. Any answer given will open up new questions, which can also be scientifically pursued, but again not to the satisfaction of a complete answer, and so on, ad infinitum. We will call this idea the principle of theoretical incompleteness. With openings continuously available, there is always room for invoking a religious explanation. This is what we meant earlier when we said that the question of

\footnotetext{
${ }^{8}$ A hint of such a theoretical direction can be found in the quantum event interpretation (Hughes 1989), where the notion of property is supplanted, and in the relational interpretation (Rovelli 1996), where the notion is contextualized relative to an observer or system. See also Mermin (1998). These ontological difficulties have also compelled many to re-examine the nature of scientific explanation, itself (Hughes 1989, 296-319; van Fraassen 1989, 1991; Bitbol 2001).
} 
whether we can achieve a complete physical theory is only apparently different from the question of whether physical theories have any bearing on religious belief. Faith and doubt, whether of a religious, scientific, or any other kind, is an implicit personal admission of the inherent incompleteness of our ideas about the world. This is why the theist insist that he can raise his questions even in the face of a purported TOE. If the TOE was truly a complete theory, the theist would not be able to raise his questions. They would all be answered by the TOE. A complete TOE is incompatible with the notion of God. We can see this even clearer by comparing God and the TOE.

By our chosen definition, God is an omnipotent being. Any experiences we may encounter can be explained by God. "She" is almost like an ultimate scientific theory. It is as if we said the universe is made up of a single ultra-particle that can manifest a myriad of properties and is responsible for a myriad of interactions. In a way, this is what a TOE is after; in fact, there is a hypothesized particle, the Higgs boson, that has been bestowed this almost God-like property. In essence, God is like a TOE and a TOE is like a God. We have to pick one of the two; we cannot have both. It is this lack of awareness of the full import of a TOE that is at the root of crisis in many an ontological debates concerning God and science.

1.4.1. Arbitrariness. Another way to see the dilemma is in terms of arbitrariness, or the amount of assumptions one has to make for any given theory. TOEs, like other good theories, aim to be minimally arbitrary. This is why the Standard Model of particle physics, while a robust and useful catalog for the working physicist, is considered a bad theoretical description. It has some twenty arbitrary parameters that must be supplied from experimental data rather than being derived from within the theory itself. It is one of the goals of any future TOE to eradicate this sloppiness. Counterexamples to the Standard Model are Newton's theory of gravity and his theory of motion, both of which, by themselves, unified the movement of the heavens with the common objects of the earth. The same laws that applied down here applied up there, thus eliminating the need for two separate sets of laws. Einstein's theory of gravity went even further by unifying the law of gravity with the law of motion. Because of this, it is considered to be one of the most elegant and yet most powerful theories ever put forth. Quantum theory is mixed on this front. The primary reason being that there are two different laws to apply depending on the situation (nonmeasurement and measurement), without an explanation of why. The goal of an improved quantum theory or reinterpretation would be to eliminate this arbitrariness by means of a single principle that would account for both laws.

But no matter how much arbitrariness is eliminated from scientific theories, as long as some remains, there is an opportunity to raise questions. For example, in the case of Einstein's gravitational theory, one can ask, why four dimensions instead of eight? What is so special about the number four? TOEs, by aiming to be a fundamental theory, seek to eliminate such questions and arrive at the core truths. Indeed, something is fundamental exactly because it is not arbitrary or haphazard. But how far must we go in order to eliminate arbitrariness? How far till we can account for four dimensions? Till we can account for force? Till we can account for an atom, a nucleus, a quark, a string, or space itself? How far do we need to dig to get the fundamental answer(s)? Can any physicist provide a reasonable answer to this question? Once again, we find that there can be no natural ontological criteria where we can draw the line. A purported TOE or any other scientific theory contains some amount of arbitrariness, some set of assumptions upon which the theory is built, and this arbitrariness will leave it vulnerable to questioning. We will discuss arbitrariness more thoroughly in Part II when we consider its more formal manifestation within the philosophy of science, the Quine-Duhem thesis.

1.5. The Criticism from Finite Experience. At this point it is necessary to address a major criticism that the above philosophy faces. Some may contend that there does exist a natural ontological criteria that distinguishes scientific questions from non-scientific ones, and that demarcate the line between fundamental physical notions and arbitrary ones. This contention 
rests on the notion that our experience of nature is finite or ultimately reducible to atomic components. This notion is espoused in Hawking's earlier view $(1988,167)$ that physics stops at the Planck energy limit, that it will be physically impossible to probe nature past this energy barrier-incidentally, this was one of the reasons he was advocating a TOE, a position which he has since somewhat backed away from (2002). Though we have yet to realize any such limit, if it should exist, then we would be correct in saying that some questions are beyond science, since we have no way of experimentally examining it. Whatever entity we observe as our atomic experience (e.g., at the Planck energy level) would be the fundamental entity of our TOE. This would allow us to eliminate arbitrariness and formulate a complete TOE, while also permitting us to believe in a heavenly deity. However, we will shortly see that a key component of our argument, which we have yet to expose, forbids us from subscribing to such an ideal position.

If we are then to reconcile with our original notion of theoretical incompleteness, we have to reject the notion of finite or atomic experiences. We thus adopt the principle, herein referred to as experiential incompleteness, or the novel experience problem, which states that our experiences of any given phenomena are incomplete and augmentable. This is not purely an external characteristic of nature, but is inherent to our experience. It goes hand in hand with theoretical incompleteness. The same sensorial and cognitive processing mechanisms that allow us to experience and form theories of nature also preclude these experiences and theories from being complete. Experience and understanding, however sophisticated, are imperfect luxuries of cognition. An immediate and obvious example of experiential incompleteness is the precision gap that is accompanied by any physical measurement. Experimental measurements are never 100\% precise; there is always room for improvement. It is in this room where novel experiences lie. In Part II we will take a closer look at this and other forms of experiential incompleteness.

1.5.1. The Apparentness of Convergence. The unawareness of the novel experience problem is also responsible for the illusion of convergence, the idea put forth that all our empirical evidence seems to be converging toward some final theory. If all there is the current empirical evidence for some domain of phenomena, then we are right in saying that they converge on some final theory for that domain, barring the Quine-Duhem thesis (as to be discussed in Part II). The proponents of convergence, of course, recognize that for many domains of phenomena, there may still be some additional empirical evidence yet to be discovered, but they insist that the new amount will only be a fraction of the current evidence, and therefore their effect will be negligible on the current theory. What we must keep in mind is that at some point in the future, today's empirical evidence will only be a fraction of the then current total of evidence, and who knows what theory this running total will converge towards. The point is that convergence seems so only on hindsight.

Though we cannot make a case for the future, the history of science supports this viewpoint. A textbook example occurred at the turn of the last century, when physics was almost wrapped up, save two experimental anomalies: black-body radiation and the lack of detecting the ether. The first led to the discovery of quantum mechanics and the second to that of the special theory of relativity. Compare the amount of empirical evidence gathered since then with what existed before.

Of course, many feel that for fundamental physics it is different this time. The Planck limit seems unlikely to be penetrated. But is it really fair to make a judgment about a possible barrier that presently is so far off from our experimental reach? There is a vast energy difference between the level of our current particle colliders $\left(10^{4} \mathrm{GeV}\right)$ and the Planck energy $\left(10^{19} \mathrm{GeV}\right)$. Additionally, to imply that any experimental discoveries made on the long road to the Planck energy will also converge toward our current theories is highly presumptuous. Already, experiments coming out of some of the newer and upgraded facilities are beginning to strain the Standard Model of particle physics (Choi 2003; Evdokimov 2004). In Part II when we discuss the novel experience problem in detail, we will provide additional examples of empirical discoveries that challenge current theoretical models. 
1.6. The Apparentness of Non-Scientific Questions. By our thesis, all questions about our phenomenological experience are amenable to scientific inquiry; nonetheless, it seems that some are far removed from the domain of science. Before coming to the coda of our argument, we pause to address this apparent difference. By looking at our debated list of TOE requirements (Section 1.4), we see that the first question is obviously regarded by scientists and theologians alike to be an issue for science, but that as we progress down the list, this appears to be less and less the case; however, at the same time, we cannot think of any scientific or logical reason that would prevent us from theoretically or experimentally pursuing an answer to all of them. The source of this disparity in conviction then lies in the following. The first question seems readily to be one for science because there is an active and fruitful amount of theoretical and experimental work presently surrounding it; whereas we progress down the list, this becomes less and less the case. In some cases, it is not even clear where to begin an attempt, what direction the research should take.

To illustrate this point, let us consider an earlier phenomenological question that is not on our list: How was the universe created? Prior to the twentieth century, such a question would have been considered almost solely the domain of religion. Scientists were hardly speculating on the issue, if at all. Their central concern was figuring out how the universe worked; they could not even begin to imagine from whence it came. It was just assumed that it was always there, perhaps placed by God. It was only after further astronomical observation, combined with theoretical insight from Einstein's theory of gravity, revealed that the universe was actually expanding that the question began to be considered scientifically, resulting in a tentative and still developing answer: the big bang hypothesis. Before these theoretical and experimental developments, there was simply no scientific framework in which to embed the question. A very similar parallel can be found in how the question of human creation turned from a religious one to a scientific one via Darwin's research. In this light is it fair to say that the question of whence the big bang itself came from will never be considered a question for science?

As another example, consider question two on our debated list of TOE requirements (Section 1.4), regarding whether the physical constants and parameters are uniquely determined. After much research into string theory, the possibility began to emerge that the theory may not uniquely predict these values. It was unclear how to go about finding a solution. This lack of direction led many to doubt whether a scientific answer could ever be found. However, with the suggestion that quantum mechanical probability amplitudes may play a role, renewed scientific interest flourished once again in the question.

As a final example, consider the questions surrounding quantum physics. When quantum theory first appeared on the scene, questions about superposition, the measurement problem and non-local correlations were strikingly novel. At that time there was not even a clear formulation of the questions, due in part to the bizarre and mysterious nature of these phenomena. This ignorance was also kept on by the fact that the very success of quantum theory drew attention away from these foundational issues and towards the more productive application of the theory. Nonetheless, some physicists did work on these questions, with more joining in as time went on, and today we see that the questions once thought unapproachable have begun yielding to theoretical and experimental investigation, as outlined in Section 1.3.4.

These examples follow the pattern of paradigm evolution and revolution as elucidated by Kuhn (1970). When a new theoretical paradigm answers the questions of an older paradigm, much investment is spent by the scientific community in explicating and applying the new theory to ever broader phenomena, until such application meets with resistance and questions concerning the new theory itself begin being investigated, and the cycle begins once again. The distant fuzziness of the questions that lie on the periphery of our knowledge appears inconsequential to the grand theoretical edifice presently in front of us. It seems only natural that they can be dismissed as unscientific or of only minor concern. In this regard, an earnest scientific investigation into questions three and five on our list of TOE requirements 
(Section 1.4) will not begin until when (and if) we have a successful string theory and a more thorough account of the big bang, respectively-although some have already begun speculating on these issues. We also note that a resolution to any of these questions does not necessarily lie in a revolutionary new theory. In many cases it is not clear till the end whether we need a new theory, more ingenuity in the application of our original theory, or even an addendum to or reinterpretation of it.

1.7. The Central Problem: Self-Reference and the Undefinability of Valid Observations. Incompleteness, the novel experience problem, belief, arbitrariness, and the inability to find any natural ontological criteria to distinguish science from religion all arise from a more central problem. To see this, imagine how the debate over God's existence would turn out if we were to attain a TOE. It happens that such a state of affairs would be somewhat paradoxical for the debate. For we need a TOE in order to test for any miracle observations, but a TOE, by our given definition (Section 1.3), should ultimately account for all our observations. Nonetheless, we can suppose that once we do achieve a TOE, then we have scientifically ruled out God, but if at any time we observe some miraculous phenomena, then we are willing to acquiesce that a God exists. Now, however, we are presented with a new problem: how do we know that the observation in question is truly a miracle or a natural observation that disconfirms our TOE?

To this extent consider how we would empirically determine whether any given TOE is the correct one. If we follow Popper's (1959) definition, then the theory must be falsifiable by observation in order to be empirically tested. ${ }^{9}$ Thus there must be a set of observations that the TOE predicts and a set that it does not. Say, hypothetically, we observe one of the observations that it does not predict. This would seem to indicate that the theory is incorrect. But of this observation we can ask how do we know whether it is sound?

It is here that the notion of valid observations, as mentioned in the main introduction, becomes relevant. Why should we ask whether our observation is sound or valid? Is it not obvious? For example, the lead ball fell down with a specific acceleration, or the electron was deflected at a specific trajectory. But it is not always obvious, and difficulties do abound, as pointed out in the main introduction. Moreover, what if someone insists that the observation did not occur so? By what authority is she renounced as wrong? Popular consensus? No. If we are to be scientific about it, then we should ask for some scientific procedure that can establish whether the observation did indeed take place.

What sort of procedure would this be? Simply, it would be a procedure based on a scientific theory, a theory about the sensorial-cognitive interactions of a human being with his environment, a theory upon which when certain observable conditions occur within the sensorial-cognitive system of the human being, it can be said that the human being has indeed experienced a certain observation. We will call this theory, "the theory of valid observations."

We will now show that such a theory cannot exist, and hence demonstrate one of our central results: the theorem of undefinability of valid observations. At this point, the route of our argument depends on what we consider to be the domain of a TOE. If we go with our prior assumption of Section 1.3, in which science is reductive and that the TOE can account for processes beyond the domain of physics proper, including the behavior of complex biological entities and, specifically, the sensorial-cognitive behavior of human beings, then the TOE should contain the theory of valid observations within it and therefore can tell us whether our observation is valid. But this puts us in an impossible situation: in order to determine that the observation that falsifies a TOE is valid, and hence that the TOE is falsified, the TOE-which includes the theory of valid observations within it-cannot be false. This contradiction tells us that even if a TOE can discourse about our sensorialcognitive behavior, it cannot go so far as to tell us whether our experienced observations are

\footnotetext{
${ }^{9} \mathrm{We}$ only wish to follow Popper in spirit here. It is not necessary to adhere to strict falsificationism, but only that we allow observations to arbitrate in some way on scientific theories.
} 
genuinely valid, lest it becomes inconsistent. A TOE cannot contain the theory of valid observations within it.

If, on the other hand, we assume a TOE cannot (or should not) account for sensorialcognitive processes, then it cannot discourse about our observations, and hence, a fortiori, is unable to determine whether our observations are valid. But then in such a case we can ask whether the theory of valid observations exists independently of a TOE? By similar arguments above, we show that such a theory cannot exist.

Assume that it does exist. Now recall, as in the case of a TOE, that the observational predictions of a scientific theory, in general, fall into two categories: observations that corroborate the theory and ones that falsify it. To our theory of valid observations we put the question: Is an observation that falsifies the theory, itself, a valid observation? Assume it is. This then implies that our theory of valid observations is falsified. But this in turn implies that we are no longer certain that the falsifying evidence is valid, which further implies that we are no longer certain that the theory is in fact falsified. ${ }^{10}$ This is similar to the result we got above on the TOE. Both generated contradictions. These contradictions tell us that a TOE complete enough to guarantee the validity of our observations or a theory of valid observations, itself, cannot be both empirically falsified and consistent, and therefore cannot be consistently empirically tested; both are chimeras. Now, of course, a more restricted theory of observations may exist, but it will be incomplete and cannot guarantee the validity of our observations.

This is precisely why a TOE leads us to the paradoxical situation in our empirical debate over God's existence. Only a TOE complete enough to guarantee the validity of our observations can be used to test for God's existence, but such a theory cannot exist. A TOE or any other theory cannot guarantee the validity of our observations nor disprove whether they are miracles. This is why the theist is able to raise his doubts concerning the impregnability of a TOE, and this is ultimately why the debate over God's existence is empirically undecidable.

The inability of a TOE to secure the validity of scientific observations is core to our arguments. Without a theory to tell us what constitutes valid empirical data, we are unable to draw a clear boundary encompassing the hard facts of science. This failure undermines our ability to find any natural ontological criteria to distinguish questions of phenomenological experience into scientific and religious (or other) categories, or demarcate fundamental physical notions from arbitrary ones. At the same time, it undercuts the notion of a finite (or atomic) experience set. As will be seen in Section 2.3, if experiences were finite, then we could determine their validity.

With the theorem of undefinability of valid observations, we can assert a general principle of scientific theories: no empirically testable theory (TOE or otherwise) can guarantee the validity of its observations. Lacking such a guarantee, we cannot then absolutely say whether some observation actually confirms or disconfirms a theory.

These last two statements may lead one to wonder (1) how is it that at many times it seems that some definite observation has taken place, and (2) how can science ever make any progress? The first question is answered in Section 2.3; its resolution lies in the principle of experiential incompleteness. The second question we answer now.

Before Popper (1959), philosophers and scientists assumed that theories can be confirmed or disconfirmed by empirical evidence. Popper argued that this was a naïve viewpoint, in that experiments cannot confirm a theory, but only corroborate it. Nonetheless, Popper still held to the conviction that experiments can falsify, or disconfirm, a theory. Most scientists (if not philosophers) today hold to this position in some form, and thus science can progress without experimental confirmation, as long as it is still able to disconfirm theories. But, of course, Popper's remaining conviction of falsification was challenged by the holistic turn in

\footnotetext{
${ }^{10}$ It does not help in such an event to modify the theory or rely on some other theory of valid observations, because we can raise the same concern with a new empirical falsifier. Also it does not help to have several separate (perhaps infinite) theories of valid observations, as we would have to merely go through a larger chain of circular reasoning before arriving at the contradiction or else fall into an endless regress.
} 
the philosophy of science led by Duhem ([1906] 1954) and Quine $(1951,1960) \cdot{ }^{11}$ Undermining this remaining authority of observations leaves us in the same quandary regarding the selection of theories as our theorem above. Holists and other philosophers have responded to this quandary with various answers ranging from aesthetic, pragmatic and social selection pressures (see also Section 3.4.3). Here we respond with an answer arising from the principle of incompleteness, as follows. We pick theories based on our best theoretical and empirical knowledge at the time. Such knowledge may at times seem so definite as to determine a unique theory, but as both theory and experience are inherently incomplete, further theoretical and empirical discoveries may reveal our judgment to be inaccurate. This answer will be expounded in Part II, especially Section 2.5.

It is important for us to understand why a TOE or any other theory is unable to secure the validity of scientific observations. Scientific theories generally talk about, or refer, to the outside world, the world of observations. When we ask the theory to validate those observations, and thus examine the process by which we make those observations-this would include everything from our instruments to our senses and to our final mental realization that those observations have taken place-we are turning the theory back onto itself, or more precisely, back onto us. This creates the dilemma of self-reference. However, it is unavoidable. We are a part of the physical universe that we are trying to describe. To give a complete description we have to include ourselves. Another way to see the dilemma is that a part of the universe- the sentient biological entities-is trying to describe the whole of it. The inability to extricate ourselves from this description pollutes any objectivity that such descriptions may have.

The problem of self-reference has been know to philosophers and logicians for quite some time, most notably as the liar or Epimenides paradox: "This statement is false" or "I am lying". But the issue was considered innocuous to theories of knowledge until its role in demonstrating the incompleteness of arithmetic was illustrated by Gödel (1931) in his celebrated theorem. However, even with this illumination, there was not an eye-opening realization that self-reference was a problem for our general understanding of the world; discussions of it rarely went beyond the confines of logic and mathematics. Our findings here show that the thorn that has remained in our side all this time cannot be so easily removed. A resolution to the ontological and epistemological issues facing science and philosophy cannot be had without addressing the issue of self-reference. In Part III we will take a more in-depth look at self-reference and the similarity of its role in mathematical and scientific incompleteness.

\section{PART II: PHILOSOPHICAL THEORY}

2.1. Introduction. The above analysis of the God existence debate contains some core issues within the philosophy of science whose reach goes beyond TOEs to scientific theories in general. It will be worth illuminating their role here to give us an even deeper picture of the field that we are maneuvering in.

The impossibility of an empirically testable complete TOE and the inability to resolve the God existence debate rest on one central issue: the determination of valid observations. No physical theory can guarantee the validity of observations. It is the assumption that the TOE could do so that led to the contradiction that a TOE can be empirically falsified if and only if it is not false. Like the theist, we can always ask what may lie behind our observations. Is it God? Is it some other theory? It is simply not possible to eliminate such doubts from an observation. What this means is that there is no such thing as hard observations or hard empirical data. This brings us to a well known issue within the philosophy of science: the observational/theoretical distinction.

\footnotetext{
${ }^{11}$ It should be kept in mind that Popper advocated a sophisticated form of falsificationism that was not naïve concerning holism or theory-ladenness. This, however, did not deter the continued escalation of holistic challenges.
} 
Thus far within the philosophy of science there has been no successful attempt to develop a criteria for distinguishing observational terms from theoretical ones. In addition, the field of neural science, albeit young, has been unable to clearly demarcate the line between sensorial and cognitive processes (debated in Churchland 1979, 1988 and Fodor 1984, 1988). These results are not an accident. We postulate here that sensorial and cognitive processes are fundamentally inseparable, and that it is this inseparability that is the root cause of the observational/theoretical distinction failure and the theorem of undefinability of valid observations. This postulate more clearly specifies our earlier contention that experiential and theoretical incompleteness are not purely attributable to external characteristics of nature, but are something inherent to our sensorial-cognitive capacity for experiencing and understanding nature. If we accept the insight of this postulate, we see that the following four ideas are all related to one another: (1) the theorem of undefinability of valid observations, (2) the observational/theoretical distinction failure, (3) the unattainability of a TOE and (4) the empirical undecidability of God's existence (the possibility of belief). The relations do not end there. We will argue that the following additional ideas are also related to the previous four: (5) the Quine-Duhem (and underdetermination) theses, (6) the incompleteness of any scientific theory and (7) the novel experience problem. In what follows, we will detail these ideas more clearly and spell out the relations between them all. Before continuing, we would like to caution that the above ideas are not meant to encourage an attitude of science bashing, outlandish anti-realists positions, or other philosophical anarchy. More will be said on this in Part III (Section 3.4.3).

2.2. The Quine-Duhem Thesis and the Observational/Theoretical Distinction Failure. We begin by considering the Quine-Duhem thesis, which states that any experimental data on a given domain of phenomena can be accommodated by any scientific theory purporting to describe that phenomena. This is a more robust formalization of the intuitive notion of arbitrariness that we discussed in Part I: if scientific theories are not uniquely determined, then there is some arbitrariness in theory selection. The significance of the Quine-Duhem thesis is that it asserts that scientific theories cannot necessarily be falsified by empirical evidence. We want to show that this is only possible because of the observational/theoretical distinction failure. This can be seen most simply by noting that if observation has some amount of theoretical aspects to it, then by suitably modifying those theoretical aspects we can, in essence, modify what has been observed, and thus accommodate the observation to the theory.

A more sophisticated approach is through subscribing to Quine's holistic model of scientific theories: the web of belief $(1951,1960)$. In Quine's web, scientific (and auxiliary) terms, concepts, laws and entire theories are interconnected so that their meanings do not stand on an island but depend on the meanings of other terms, concepts, laws and theories to which they are connected. In his web, Quine includes connections between observational and theoretical terms. By adjusting the meaning of theoretical terms or adding additional ones to the web of a given theory, we can impact the meaning of observational terms, and thus affect what we observe. What is essentially happening is that the observation portion is being pushed around; the theory is tailoring the observation to suit itself. In some cases, the tailoring will be minor, in other cases, major. Examples from history include Ptolemy's addition of epicycles to the geocentric model to accommodate the observed oddities of the motion of the other planets, and the inclusion of Lorentz forces to Newtonian mechanics to account for the negative results of the Michelson-Morley experiment to detect absolute space.

Some critics may raise the objection that the Quine-Duhem thesis (or a holistic model of science) does not require the abandonment of purely observational terms. They may argue that observational terms can be connected to theoretical terms without impinging on the former's sovereignty, so that the meaning of the observational terms remain fixed while the theory or theories may change around it. We will argue that such a position is untenable. We will first argue that the Quine-Duhem thesis necessarily implies that scientific theories are 
incomplete, and then argue how this incompleteness implies the observational/theoretical distinction failure.

2.2.1. The Quine-Duhem Thesis and the Underdetermination of Theory. There are two cases to consider because the Quine-Duhem thesis is a generalization of the underdetermination of theory by observation (UDT). Before we begin then, let us clarify the nature of this generalization. UDT asserts that observations are compatible with more than one theory, and therefore an observation underdetermines multiple competing theories on a given domain of phenomena. We generalize UDT to the Quine-Duhem thesis by simply replacing the phrase "more than one theory" or "multiple theories" by the phrase "any theory." The new statement, "any observation on a given domain of phenomena is compatible with any theory on that domain of phenomena" is the Quine-Duhem thesis. The Quine-Duhem thesis simply gives UDT unlimited compatibility.

The counterpart to (as opposed to the generalization of) UDT is the following: any theory on a given domain of phenomena can accommodate two or more seemingly incompatible observations on that domain. This will be correspondingly referred to as the "underdetermination of observation by theory" (UDO). This thesis emphasizes the flexibility of theories in accommodating multiple incompatible observations, while UDT emphasizes the flexibility of observations in accommodating multiple incompatible theories. We can generalize both theses to one, the Quine-Duhem thesis, by making their respective flexibilities unbounded so as to accommodate any observation (UDO) or theory (UDT). The Quine-Duhem thesis then can be seen to have two aspects: one touting the flexibility of theories, the other that of observations, depending on whether we wish to hold on to a given theory or undermine its sovereignty, respectively.

In what follows, we will make our arguments concerning UDO and UDT, the conjunction of which can be referred to as the "limited Quine-Duhem thesis." It is obvious that if the limited Quine-Duhem thesis implies incompleteness, then the much stronger Quine-Duhem thesis does too.

2.2.2. Underdetermination of Theory and Incompleteness. Does the underdetermination of a theory necessitate the incompleteness of the theory? We now argue that it does. Recall the arguments above concerning God and TOEs. There we saw that in lack of a complete TOE, it is possible for God or rival theories to compete for explanatory purview. We also saw that the success of this argument rests on the critical realization that complete theories leave no explanatory room for competing theories. That is to say complete theories explain every aspect of physical phenomena; they are not found wanting anywhere in their explanation. It is easy to see that this realization is not limited to theories of everything, but also applies to theories which may address only a specific domain of physical phenomena. (Some contend that theories of specific domains are partial to begin with and therefore incomplete, a fortiori; however, this point has also been used to mount an argument against underdeterminationsee the next paragraph.) Thus it is not possible to have two or more competing theories on a given domain and, at the same time, for those theories to be complete. Each of the theories must leave some room so that it is possible for the other theories to also be right.

We note here that incompleteness has also been used to argue against UDT. Hoefer and Rosenberg (1994), for example, point out that Laudan and Lepin's (1991) critique of UDT holds only in the case of partial, incomplete theories. ${ }^{12}$ For brevity, we will crudely simplify the extensive and detailed arguments of the above authors: present incomplete theories are subject to future change, and thus two incompatible theories that may be empirically equivalent presently, and therefore underdetermined, may not be in the future. ${ }^{13}$

\footnotetext{
${ }^{12}$ Hoefer and Rosenberg claim that theories of specific (localized) domains of phenomena are such theories, but their argument would also hold for (global) theories of everything that are incomplete.

${ }^{13}$ The same argument can be made, as Laudan and Lepin originally did, without strictly requiring theories to be incomplete, but only that they be attached to auxiliary or background theories, which may change or be replaced, depending on future researchessentially Quine's web of belief. Though, in the case of global theories, incompleteness must be admitted, since global theories
} 
Incompleteness, or auxiliary theories, is a double edged sword: it can be used to argue for or against underdetermination (similar to how it can be used to attack or defend theories, via UDT or UDO, respectively-as we will shortly see). However, while the incompleteness arguments of the above authors allow for the possibility that certain rival theories on a given domain may not be underdetermined in the end due to potential future changes, unless the incompleteness is eliminated altogether from the current reigning theory, there is the possibility that some other theory may be correct. And it is in this sense that we argue that the underdetermination of a theory is made possible by its incompleteness. ${ }^{14}$

2.2.3. Underdetermination of Observation and Incompleteness. Now we turn to the other half of the Quine-Duhem thesis. Does the fact that a given theory can accommodate multiple, seemingly incompatible empirical evidence require that the theory be incomplete? To answer this, let us consider a specific example. How was Ptolemy able to hold on to Aristotle's geocentric model in spite of the anomalies of the planets' motion that was observed at the time? Obviously he introduced the mechanism of epicycles into the simpler geocentric model. But why was he able to do this? It is precisely because the notion of a circular orbit was not defined enough in the original model. In that model planets travel in perfect circles around the earth. In Ptolemy's modifications, the planets still travel in perfect circles around the earth, except now there is more than one circle per planet. If the notion of a circular orbit had been completely or even more fully defined (e.g., there can only be one circle per planet), Ptolemy would not have been able to introduce his epicycles (unless by some other convoluted route perhaps); he would have to take advantage of an alternative hole in the theory.

As a more detailed example, consider the explanation of light in terms of Newton's particle (corpuscular) theory and Huygens' wave theory. Newton was successful at explaining many phenomena of light in terms of his paradigmatic mechanical notions of particles and forces, but ran into difficulty with the phenomenon of refraction, a phenomena which Huygens' wave theory explained quite easily. To explain refraction, Newton ([1730] 1952) postulated the existence of attractive forces that act upon the light particles at the boundary between the two mediums where refraction occurs. This was already an added complication to his theory, for it is clear how a particle of light incident on a surface (e.g., a mirror) may reflect off of it, similar to how a billiard ball may bounce off the edge of the billiard table at the same angle that it strikes it, but what are the origins of an attractive force that would cause the light particles to bend away from its straight path as it enters a material (e.g., from air into water)? Nonetheless, such an explanation would not seem necessarily outlandish in Newton's time, for there was hardly a thorough understanding of how light interacts with matter; the gap in knowledge at the time could easily accommodate any such explanations. ${ }^{15}$ However, the story is not over. In his own prism experiments, Newton discovered that not only did light bend as it enters materials, but that different colors of light bend at different angles, thus separating out the colors of white light into the well-known rainbow. To explain this, he postulated that light particles possess some additional property which determines how responsive they are to the previously postulated attractive forces (Hendry 1980; McGuire 1983). For example, a blue light particle, possessing more of this property is deflected at a greater angle than a red one, possessing less. Once again we find that, even though Newton was ultimately unable to give any definitive account of this novel property, a lack in understanding of what a light particle was at the time would certainly permit the possibility that it could exhibit such a property. The lesson to be learned is that the particle theory of light and its associated web of belief - a mixture of Newton's mechanical

by definition include all auxiliary and background theories. Indeed, as it has already been stated and will become clearer later, incompleteness and the need for auxiliary theories are intimately related.

${ }^{14}$ Interestingly, the proponents of underdetermination, to undercut this argument against it, have moved the debate to the arena of complete (global) theories. We, of course, are arguing here that by giving up the sword of incompleteness, the proponents have no fight.

${ }^{15}$ For example, Newton postulated that light particles experience a gravity like force near the surface of the material. For a more thorough account of these explanations, see the citations in the referring paragraph. 
and gravitational theories combined with alchemy and religion-was never complete in its description of light and its interactions with matter, nor could it answer all questions concerning the behavior of matter in general. And it was therefore possible that the yet unknown theoretical descriptions could account for some new, seemingly anomalous observation. In this way Newton's particle theory of light lasted for another hundred years.

As enduring as the particle theory of light was, further complications in the theory eventually gave way to the simpler wave theory. The wave theory faced its own trials at the turn of the twentieth century, and in an ironic twist of events, we get another example of theories being modified. The observation of the photoelectric effect, where light seems to be absorbed and emitted by matter in discrete chunks as if they were particles, caused renewed interest in the particle theory. At this point, the proponents of the wave theory introduced a modification that would allow their theory to also compete as an explanation: the wavepacket. Light is still a wave, but the amplitude of a given light wave smoothly diminishes at a given distance from either side of the central wave peak. This modification extends our notion of waves while still being compatible with its central ideas, albeit complicating it somewhat. Here too we find that the modification was only possible because the theory of light waves was incomplete. The theory never specified all the different ways in which a light wave can vibrate. Indeed, going further, can we ever give a complete formal definition of what constitutes a wave in general? For example, is a square wave actually a wave, even though it changes its amplitude discontinuously? What exactly qualifies something to be a vibration? How do we exactly define amplitude and wavelength? It is the contention here that such concepts cannot be given a complete definition. This applies to all physical concepts, whether wave, particle, force, orbits, electrical attraction, etc.

It is this incompleteness of definition that allows theories to be saved. When faced with some seemingly anomalous observation, we go in search of answers in the fuzzy, outlying areas surrounding our theoretical concepts. There we have some room to maneuver, some yet unexplored vistas that may afford us a solution to our crisis, a place where we can further mold and shape the terrain of our definition to suit our needs. Ignorance becomes a virtue instead of a vice, and flexibility becomes more valuable than stability. With this insight we see that theories can be modified when there is some room to maneuver within the theory, i.e., if there is some explanatory room. This is consistent with the critical realization we recalled above: complete theories leave no explanatory room for competing theories, even if some of those theories are modifications of the original theories. The conclusion is that complete theories cannot be modified; only incomplete ones can.

Without going into details, we briefly list some additional examples from the history of physics, which may be followed up in the accompanying citations: the many modifications of Newton's mechanical paradigm to account for electromagnetic and light phenomena before the advent of relativity and field theories (see Einstein [1938]1966, 62-122 for a summary); an account of the perihelion advance of Mercury's orbit within the traditional Newtonian theory of gravity versus the modern account of Einstein's theory (Hall 1894; Campbell 1983); dark matter, dark energy, and other proposals to account for the difference between what Einstein's theory of gravity predicts and some of the presently observed motions of galaxies and the rate of expansion of the universe itself (see van den Bergh 1999 and Caldwell 2004 for a summary); and finally, the many hidden variable theories which attempt to save the classical deterministic paradigm in the face of modern sub-atomic observations (see Section 1.3.4 for references).

Some may contend that in certain cases above, rather than modifying existing concepts, merely new ones were added, and thus we do not require concepts to be incomplete for UDO to be successful. Even if we allow this caveat, we do, nonetheless, require the theory to be incomplete, since we earlier established that complete theories are maximally explanatory, and therefore leave no room for modifications or additions. ${ }^{16}$ So whether we talk about

\footnotetext{
${ }^{16}$ But alas, this caveat does not hold. Concepts cannot simply be added to theories without affecting the meaning of other concepts within the theory. This is the critical realization behind Quine's web. Consider the case of dark matter. By adding dark matter to the web of Einstein's theory of gravity, we are extending the notion of what we mean by mass. Previously, our
} 
extending concepts or adding new ones, this is only possible if the existing theoretical framework is incomplete.

We do not wish to give the impression here that incompleteness is the sole reason why displaced theories have lasted as long as they have. Newton's mechanical paradigm, for example, was very successful in accounting for many phenomena and therefore has earned a certain level of credibility with scientists. In addition, social, religious, aesthetic and other influences may contribute to a theory's credibility. Naturally, how much anomalous evidence is needed before we are willing to abandon a theory depends on how much credibility the theory has with us. But when faced with any such seemingly anomalous evidence, whether small or large, it is incompleteness that allows us to implicitly rationalize the correctness of a theory or, equivalently, retain our faith in it.

2.2.4. The Quine-Duhem Thesis and the Undecidability of God. Before continuing with our argument, it is worthwhile to pause and point out some interesting parallels between the God existence debate and the two aspects of the Quine-Duhem thesis. The inability to prove that God does exist can be likened to the inability to prove whether any given theory on a given domain of phenomena is the correct one. The failure is due to the underdetermination of theory. God and the purported TOEs are underdetermined by all available empirical evidence, just as the competing theories on a given domain are underdetermined by the evidence of that domain. Second, the inability to prove that God does not exist can be likened to the inability to prove whether any given theory on a given domain of phenomena is not the correct one. This failure is due to the underdetermination of observation. We can modify our notion of God or the theory to accommodate any empirical evidence, since neither God or the theory are completely defined. These connections throw light on ideas that have always been an integral part of science but has traditionally remained obscured: doubt and faith. The underdetermination of theory allows us to doubt scientific theories, and the underdetermination of observation allows us to have faith in them. In this respect we see that science and religion share a certain bond.

2.2.5. Incompleteness and the Observational/Theoretical Distinction Failure. We now continue with our argument. We have argued that the limited Quine-Duhem thesis, and thus the Quine-Duhem thesis, implies that physical theories are incomplete. We now argue that this incompleteness necessarily implies the observational/theoretical distinction failure, and thereby try to demonstrate that the success of the Quine-Duhem thesis indeed rests on this failure. The argument is straightforward. The incompleteness of physical theories means, as given by our definition of completeness in the main introduction, that there are questions about the domain of phenomena under consideration about which the theory is unable to answer. This means that we cannot completely explain every aspect of a domain of observations; moreover, we cannot completely explain every aspect of any single observation. If we could in either case, then we would have a complete theory accounting for the domain of observations or for the single observation. (In the case of a single observation, we might be more inclined to call the theory an explanation or even a description, but the point is the same: the description, explanation, account or theory is incomplete.) Whether for a single observation or a group of observations, if the Quine-Duhem thesis holds, so does an incomplete account of the observation(s). The core argument here is that if a theory is sufficiently incomplete to accommodate more than one consistent and maximum set of observations, then it is incomplete in its description of any such set of observations.

If the description of an observation is incomplete, then we cannot give a complete list of conditions that must obtain in order for us to say that the observation has taken place. We

definition of mass included only the mass particles that constitute the standard model of particle physics, but by postulating dark matter, we are saying there may be new kinds of mass particles, for example, what some physicist have termed the weakly interacting massive particles (WIMPs). In Newton's explanation of refraction, the postulation of "attractive forces" impacted the notion of gravitational force, or force, in general. Thus additions to theories can equally be interpreted as extensions of existing concepts. 
therefore cannot definitively determine whether the observation has taken place and thereby utter the appropriate observational term signifying our recognition of this observation. This undermines our ability to assign unique observational terms to observations, hence the observational/theoretical distinction failure. This result is also clearly consistent with the theorem of undefinability of valid observations.

Another way to see this is to note that if the description of an observation is incomplete, then we can extend the description in a new way. This new description will also be a description of the observation, albeit different. The meaning of the observation has changed in a certain sense. This mutability is the chief import of the observational/theoretical distinction failure. The meaning of the observation is dependent on how we describe it or, equivalently, the theoretical constructs we surround it with. We can paint the observation in a variety of ways and thus make it part of many different theories. The observation has no inherent meaning in itself; its meaning arises within our language, description or theory.

What we essentially have here is the notion of an observation as a cloud of theoretical constructs in which layers can be peeled, replaced, or further added to reveal or bring out different "observations." New theories reveal the theoretical assumptions that were an implicit part of the observations of prior theories. For example, Copernicus' theory revealed the implicit assumption that the earth stood still in observations of the retrograde motion of heavenly bodies; Galileo and Newton's law of inertia revealed the assumption of frictionless motion; special relativity revealed the assumption of rigid measuring rods and ideal clocks; and quantum mechanics revealed the assumption of interaction (disturbance) free observations. Such peeling or revelations are possible because the meaning of the theoretical constructs surrounding the observation are not completely defined. This incompleteness is what also precludes the observations from having definitive language terms assigned to them. Language cannot completely capture observations, and therefore, by conceding to use language to describe observations, we must allow for flexibility and mutability in its usage.

Within the philosophy of science there has always been a suspected link between the observational/theoretical distinction issue and the Quine-Duhem thesis, but it has not always been clear whether this was the case and what the nature of the suspected link was. What we see here is that there is a definite link: incompleteness. The underlying success of the QuineDuhem thesis is due to the fact that the meaning of individual terms in a theory are incomplete, thereby allowing the theory to be adjusted to accommodate any data. This is ultimately the grounds for a holistic model of science. There is no reason to link together terms whose meanings are completely defined. Holists acknowledging, at least implicitly, that the latter is not possible, recognize that terms must draw on other terms for further support.

But this does not mean that the meaning of any term, though not contained in itself, can be contained in a web of terms. No word or even a group of words can completely capture the meaning of observational terms. Such is the whole point of the Quine-Duhem thesis: a theory can accommodate any data, not just some finite range of data. Traditionally it has been argued that the Quine-Duhem thesis implies that no crucial experiment can debunk a given theory because the theory is part of a larger web of other terms and theories. What is important to recognize here is that a crucial experiment cannot debunk this larger web either. Holistic models do not merely grant scientific theories some finite range of flexibility, but an unbounded range (see, however, Section 3.4.3).

2.3. Incompleteness of Observational Descriptions. How exactly are descriptions of observations incomplete, and why is it that we are unable to definitively determine whether some specific observation has taken place and, thereby, also unable to determine whether it is valid? For example, it seems that there is a tree standing in front of me, and therefore I am right in uttering the observational term "tree". What we need to keep in mind, however, is that this is more of an agreement than a simple truth. We all agree that there is a tree standing in front of me, thus making the statement appear true. Do any of us, however, have enough of an understanding of the observable tree so that we are able to distinguish all 
observable phenomena into trees and non-trees? For example (and casually speaking), is some observed large bush perhaps a tree? I may say yes, but you may say no, depending on the differences in definition that may arise beyond the borders of our common agreement and within the fuzzy incomplete areas. Moreover, is something that we all agree to be a tree really a tree? For even our agreed upon, common definition of a tree will be incomplete, and therefore we will face questions as we conduct further observations on our tree. For example, a tree has leaves. We need to make sure our tree's leaves satisfy the botanical definition of what a leaf is-so that we are not fooled by some plastic imitation tree or an even finer modern technological replica. But this definition itself is constituted of elements which have further definitions, and we will need to make sure the elements of our tree's leaves satisfy these definitions, and so on.

The above analysis can be carried out for all observable phenomena ranging from biological species down to chemical, atomic and subnuclear elements and all the way up to astronomical objects such as planets, stars and galaxies. For example, how much genetic variation is allowed before we can distinguish between biological species? A definition based on sexual compatibility cannot be sufficient unless we can offer a complete definition of sexual compatibility itself. What about atoms? Is an isotope an atom? Most would say yes. Is an antimatter "atom" an atom? What about the possibility of atoms made out of some of the many other discovered nucleons? Is a neutron "star" a star? What about the forms of matter such as solids, liquids, gases and condensates? Can we clearly distinguish all such cases? There is currently much research in this field (Paredes 2004; Plazanet 2004). Even in the case of the current "fundamental particles", are we able to define their parameters enough so that when we observe readings in our particle detectors, we can clearly identify all cases which are, say, electrons? This is not possible as of yet because, for example, in the case of the electron, we do not know how it exactly behaves at higher energies and field strengths. This question is part of a current program of particle research (Anderson 2001).

Part of the reason we are unable to definitively identify observations is due to the inability to completely define observational terms within language. For any observational term, we will find that there are cases which require us to further refine our definition in order to determine whether the observation has obtained or not. We may be led to believe that we can avoid such infinite regress in the definition of observational terms by directly associating the terms with experience, i.e., an ostensive definition. This is in the spirit of Wittgenstein's picture theory of language: we give meaning to a term by uttering it and then pointing to a phenomenon. Say, in such a manner, we assign the term "ugg" to the phenomenon of lightning strikes. The problem with such an approach is that there will be cases where we are not sure whether to utter "ugg", for example, if we observe the phenomenon of ball lightning. Moreover, with such an approach, we cannot communicate with one another the intricacies of observations; we cannot communicate similarities and differences. We cannot create the notion of common properties that many phenomenon in this world share, such as round, soft, shiny, jagged, etc. Thus, while we can say that we just experienced "ugg", we cannot tell someone what that is. We must rely on the fact that they have also experienced "ugg" and therefore know what we are talking about when we utter the term, but we cannot proceed to describe it to them. As soon as we do, then we end up with the problem of infinite regress that we had above.

With Wittgenstein's picture theory of language, we see that we avoid the problem of infinite definitional regress, but still have the central problem of undecidable observations. There may, however, appear to be a way out of the dilemma. One may contend that the problem above arose because we chose a complex observation such as lightning which has several processes going on. The picture theory of language will work if we can identify atomic, irreducible observations that are the components of the larger, complex observations. This would in principle eliminate borderline cases: either something or some process is observed or it is not. This points to a reductionistic view of science in which there are fundamental physical entities and processes. (In fact, this is what TOEs promise.) It also points to a finite view of natural experience. It is because of such finiteness that physical 
theories, being finitely described, can ever hope to completely capture our experience of nature. It is also such finiteness that any possible theory of valid observations rests on.

But we see from our interaction with nature that such finiteness does not in fact exist. We can always probe nature deeper and deeper. This capability becomes obvious in any scientific experiment, where the precision of measurements can be improved-maybe not with today's technology, but certainly with tomorrow's. By increasing the precision, we are zooming in and getting a more detailed description of the observation. For any scientific predicted result, the corresponding observation will be accompanied by a percentage error of measurement uncertainty. It is in this uncertainty that we find the undecidable cases of a given observation and further refine our observational definitions-the infinite regress. Whether a tree, an atomic nucleus, or a galaxy of stars, we can always ask for a more detailed picture, and we can get it by probing nature more deeply.

Cases from the mathematical sciences, like astrophysics, elucidate this point effectively. Consider the motion of the planets around the sun. We can describe their orbits by finite mathematical descriptions. Initially, Copernicus described them as circles. This appeared to match what little data we had at the time and seemed like a good guess. With the arrival of the telescope, we were able to get more precise data, which prodded Kepler to describe the orbits in terms of ellipses. Then in the 19th and 20th century, with even more powerful telescopes, we saw that the ellipses precess, ultimately supporting Einstein's theory of gravity. What this shows is that finite mathematical descriptions do not fully capture observations. By improving the precision of the observation, we invite the possibility of new mathematical models. This implies that in the future there may be a new mathematical model of planetary orbits or some new theory that may supplant the notion of planetary orbits. The latter is possible because, as we improve our precision, we may find that the data no longer supports the notion of planetary orbits, but rather reveals planetary orbits to be some other existing or novel phenomena. In general, we may expect improved measurement precision to sharpen, modify or even supplant prior observations.

2.4. Novel Experience Problem. This aspect of scientific experience, observational precision, we will fold into what we shall call the novel experience problem and give it a central role in our philosophy. What it states is that we can have new experiences of any given phenomena. In this way our experience of nature remains incomplete. (We recall, once again, that novel experience is not synonymous with anomaly, as pointed out in the main introduction.) Besides precision, there are other aspects of an observation that can be tuned to give us a new look at the phenomenon under consideration. We loosely identify three such other aspects: perspective, interaction and range. Before turning to them, we will first discuss the issue of precision more thoroughly.

2.4.1. Precision. It is often remarked how highly accurate our current theories are. For instance, in his popular exposition of quantum electrodynamics (QED), the quantum theory of the electromagnetic interaction, Feynman $(1985,7)$ has pointed out that the theory has been successfully tested to a precision equivalent to measuring "the distance from Los Angles to New York to ... [within] the thickness of a human hair." Let us explicate this statement more quantitatively. The quantity being measured is the anomalous magnetic moment (AMM) of a free electron. Feynman quotes the theoretical value to be 0.001159652 46, with an uncertainty of 20 in the last two decimal places. From these numbers, we can calculate the precision of the theoretical prediction: 17 millionth of a percent, or equivalently, $0.17 \mathrm{ppm}$ (parts per million). Feynman quotes the experimental value to be 0.00115965221 with an uncertainty of 4 in the last decimal place, corresponding to a precision of $0.03 \mathrm{ppm}$. Subtracting the theoretical and experimental values gives a disagreement of 0.00000000025 , with a joint uncertainty of 20 in the last decimal places, corresponding to an accuracy between theory and experiment of $0.22 \mathrm{ppm}$ with a joint uncertainty of $0.17 \mathrm{ppm}$. Although the disagreement lies outside the joint uncertainty, it represents only a 1.2 standard deviation, considered to be in good agreement. The question we raise here is: does an 
agreement at such a high level of accuracy make it unlikely or even rule out that a more precise experiment will reveal a deviation from theory?

To this extent, consider the measurement of the AMM of the muon, a heavier sibling of the electron. But first, it is necessary to give some background concerning QED and the theoretical calculation of the muon's AMM. From experiments, it is known that for larger masses and energies, such as in the case of the muon, the electromagnetic interaction interacts with two of the other fundamental interactions (or forces) of physics, the weak and the strong. These interactions are beyond the scope of QED. It is therefore not the whole story when it comes to the electromagnetic interaction; in order to address the electromagnetic interaction at higher energies, QED must be extended. This has been accomplished for the weak interaction (the electroweak theory), but it remains one of the outstanding problems in fundamental physics for the strong interaction; moreover, the strong interaction is not that well understood. Part of the goal of any unified theory (e.g., a TOE) is the integration and better explanation of these interactions. The situation as it currently stands is known as the Standard Model. Though physicists acknowledge that it is incomplete, it is based on a certain theoretical foundation. It is this foundation whose experimental accuracy is being examined here.

Let us begin. (For brevity, the remaining numbers are given in scientific notation, the uncertainty is indicated by " \pm ", and the precision or accuracy is in parentheses immediately following.) Though more difficult to measure than the electron, by 1999, the world experimental average for the muon's AMM (based on work done at CERN and Brookhaven National Laboratory) was $(1165920.5 \pm 4.6) \times 10^{-9}(3.9 \mathrm{ppm})$ (Brown 2000), with the theoretical value, as predicted by the Standard Model, at $(1165917.68 \pm 0.67) \times 10^{-9}(0.6 \mathrm{ppm})$ (Miller $2002)^{17}$, giving a disagreement of $(2.8 \pm 4.7) \times 10^{-9}(2.4 \pm 4.0 \mathrm{ppm})$, a 0.6 standard deviation, the disagreement being well within the joint uncertainty. We already see here that theory and experiment are accurate to $2.4 \mathrm{ppm}$ - only an order of magnitude less than the electron's case-and found to be in agreement. By 2001, due to ongoing work at Brookhaven, the precision of the measurement was significantly improved, albeit not greatly affecting the value itself. The new world experimental average was $(1165920.3) \pm 1.5 \times 10^{-9}$ (1.3 ppm) (Brown 2001), with the theoretical value remaining the same. The new disagreement was (2.6 $\pm 1.6) \times 10^{-9}(2.2 \pm 1.4 \mathrm{ppm})$, a 1.6 standard deviation, the disagreement now outside the joint uncertainty. By January 2004, the Brookhaven team had further increased the precision, with a slight change in the value: $(1165920.8 \pm 0.6) \times 10^{-9}(0.5 \mathrm{ppm})$ (Bennett 2004). At this point, comparison with theory is complicated by some recent developments. The current consensus is that there are two possible theoretical values, the "directly" and "indirectly" calculated ones. The directly calculated value is $(1165918.1 \pm 0.8) \times 10^{-9}(0.7 \mathrm{ppm})$ and the indirectly calculated one is $(1165919.6 \pm 0.7) \times 10^{-9}(0.6 \mathrm{ppm})$ (Davier 2003), giving a disagreement of $(2.7 \pm 1.0) \times 10^{-9}(2.3 \pm 0.9 \mathrm{ppm})$ and $(1.2 \pm 0.9) \times 10^{-9}(1.0 \pm 0.8 \mathrm{ppm})$, with a 2.7 and 1.4 standard deviation, respectively. ${ }^{18}$ While a 1.4 deviation is not a major concern, a 2.7 is. (It is inevitable that these theoretical calculations will be updated in the future, likely settling on a single value. We also note that the increase in deviation in the direct case is not due to the change in the theoretical value since 1999; in fact, the new theoretical value is closer to the latest experimental value than the 1999 theoretical value. ${ }^{19}$ )

\footnotetext{
${ }^{17}$ This value represents a later correction of an error (Knecht 2002) that was found in the value at the time, $(1165915.96 \pm 0.67)$ $\times 10^{-9}(0.6 \mathrm{ppm})$ (see Miller 2002 for further information and references).

${ }^{18}$ One may wonder here why the new theoretical prediction is fractured and the precision not even improved. Certain portions of the calculation involving the strong interaction is vexed with technical difficulties. This has forced theorists to approximate these portions of the calculation based on experimental data, an ongoing process that is complex and not completely understood, and therefore prone to errors even outside the meticulously reported uncertainties. (For further information, see Davier 2003 and also the previous footnote.)

${ }^{19}$ Some may feel that we should have been using these new theoretical values all along in our comparisons rather than making an historical survey. There are pros and cons to this argument, but without digressing, we point out that if we had used the new theoretical predictions in the previous comparisons, it would have only made our case stronger since the new predictions are closer to the previous experimental values than their historical counterparts.
} 
By looking at the above numbers we can see that, while the experimental precision continued to improve over the years, from about $4 \mathrm{ppm}$ to $0.5 \mathrm{ppm}$, the accuracy between theory and experiment remained roughly the same, at about $2.3 \mathrm{ppm}$ (excluding the case of the indirect theoretical calculation), thus contributing to the increased deviation between theory and experiment. So here we have a case where an agreement at an already high level of accuracy yielded when the accuracy was made even greater. It is also very possible that a more precise measurement of the AMM of the free electron will result in a disagreement with the Standard Model. ${ }^{20}$ Indeed, the latest experimental (van Dyck 1987) and theoretical (Knecht 2003) results have increased the respective precisions from 0.03 to $0.004 \mathrm{ppm}$, or $930 \%$, and from 0.17 to $0.021 \mathrm{ppm}$, or $830 \%$, while increasing the accuracy between theory and experiment from 0.22 to $0.036 \mathrm{ppm}$, or $600 \%$. Though not as large as in the case of the muon, the difference between the gain in precisions and the gain in accuracy of the electron has increased the standard deviation from 1.2 to 1.7. It is suspected within the physics community that these deviations, all together, may be hinting at new physics beyond the Standard Model, such as supersymmetry (Marciano 2003; Miller 2002).

We can make a similar case for another central pillar of modern physics, Einstein's theory of gravity, general relativity. Due to the relative weakness of the gravitational force compared to the other forces and the difficulty in isolating its effects from theirs, gravitational effects are more difficult to measure; yet, some highly precise measurements are possible. Gravity Probe B, an earth orbital space mission launched in 2004, will measure geodetic precession to a precision of $36 \mathrm{ppm}$ or more (Turneaure 2003), compared to the current $0.35 \%$ (Williams 2002). Bertotti (2003) has improved the measurement of $\gamma$, the amount of space-time curvature, from $0.24 \%$ (LeBach 1995) to $23 \mathrm{ppm}$, and it is possible that Gravity Probe B (Weiss 2003) may improve the precision by another order of magnitude. Elsewhere, the planned MICROSCOPE space mission will test the weak equivalence principle (via the observation of the relative acceleration of different masses) to parts per $10^{15}$ (Touboul 2001) compared to the current $10^{12}$ level (Baessler 1999), and STEP, a proposed mission, aims to increase the precision to $10^{18}$ level (Mester 2001). Though no deviation of these values from general relativity has been detected thus far, it is certainly possible, that as we keep increasing the measurement precision, deviations may be found. Such deviations may be indicative of competing gravitational theories, quantum gravity, string theory or some revolutionary new theory (Will 2001).

Throughout science, better and better instruments are allowing for a more precise look at nature. In astronomy, for example, more detailed observations of certain regions of space have recently prompted scientists to reconsider their theories of star formation (Cappellari 1999) and galaxy formation (Glazebrook 2004; Cimatti 2004). Continued improvement in the detail of the cosmic microwave background map, such as the results in 2003 from the new WMAP satellite, are keeping astronomers busy revising the age of the universe and the time when stars were first formed, and also speculating on dark energy (Bennett 2003). In the field of particle physics, besides the AMM, there are many other measurements that scientists are continually working to improve the precision of, such as the extremely small mass of the neutrinos, the QCD lamb shift, and the electric dipole moment of various particles, measurements that scientists speculate may lead to discoveries outside the Standard Model (Andersen 2001, Hollik 2002). (The role of the latest high-energy particle colliders will be discussed in Section 2.4.4 on range.) In the field of nanophysics, researches have been able to construct an electromechanical cantilever that is capable of measuring masses on the order of attograms $\left(10^{-18}\right.$ grams), a thousand-fold increase from the previous sensitivity, and are hopeful that the sensitivity can be increased another thousand-fold (Ilic 2004). Who knows what surprises such delicate measurements may reveal. To summarize, the highly precise measurements of today do not rule out or even make it unlikely that these measurements can

\footnotetext{
${ }^{20}$ The fact that the electron measurement needs greater precision than the muon before revealing a possible disagreement is similar to how the orbit of the outer planets needs to be measured more precisely than Mercury's before revealing a disagreement with the Newtonian theory of gravity. This indicates that parts of a domain are varyingly more sensitive to specific measurement anomalies in relation to a theory. This issue is discussed in Section 2.4.4 on range.
} 
be made significantly more precise, nor that anomalies will be found with improved precision; they do not solidify the position of any of their supported theories.

2.4.2. Perspective. The perspective is the vantage point, a position in space (or spacetime), relative to the given phenomenon. For example, we can observe the motion of the planets from earth or from an asteroid in space, or even from the event horizon of a black hole (perhaps one day). As another example, consider the field of immunology. In studying a disease, our perspective is sometimes outside the body. By bringing a tissue or blood sample under a microscope, we can move the perspective to within the body, but still outside the infected area. Perhaps one day we will have nano-robots that can go into the infected area and make observations. Different perspectives give us different pictures of the world and may even suggest one theoretical model over another.

Moreover, from a prior perspective, what was theory for one observation, may become the observation itself from the new perspective. The heliocentric theory, for example, is a way to account for the observation of the planets as seen from earth. We cannot actually see the planets moving around the sun; it is a theory that explains the data pretty well. But by shifting our perspective far outside the solar system, we can actually see the planets going around the sun, and our heliocentric theory now becomes an observation. This is yet another way to blur the observational/theoretical distinction. By shifting our perspective we can move between observation and theory.

2.4.3. Interaction. Another component of observation is interaction, the method by which we literally interact with nature: smell, sight, hearing, touch, and with technology, infrared, radio waves, X-rays, sonar, etc. In order to make any observation there has to be a physical interaction between the observer and the observed. The choice of physical interaction will, just as in the case of perspective, bring back a different picture of the phenomena under consideration.

For example, when we look at certain stars with a radio telescope, we can see that they pulsate radio waves, something that we would not see through a common light receiving telescope. If we use a UV, infrared, X-ray or gamma-ray telescope, we would see yet something different. Cosmic rays, composed of neutrinos, nuclei and other subatomic particles allow for forms of interaction besides electromagnetic radiation. What was once seen to be ordinary stars and galaxies are now seen to be novel and extraordinary stellar objects: pulsars, quasars, radio galaxies. By changing our method of interaction we have changed what we see, and what we have then seen has in turn dramatically altered our understanding of the cosmos. More recent examples of novel astronomical interactions include the cosmic microwave background radiation (as mentioned in Section 2.4.1 on precision), the gravitomagnetic field, to be measured directly for the first time by Gravity Probe $\mathrm{B}$, and gravitational radiation, awaiting to be detected by current and future detectors (LIGO, VIRGO, TAMA300, GEO600 and LISA). The later, once (and if) detected, could afford us new visions of very massive objects, such as black holes, neutron stars and pulsars.

Outside astronomy, examples include X-ray scans, which allow us to see beneath the surface of things, and electron and atomic force microscopes, which allow us to use particles of matter to see the world with greater detail than traditional light based instruments. Additionally, various forms of matter can be used to directly interact with and detect novel particles or processes, for example: water to observe neutrinos and the yet undetected proton decay (SuperKamiokande Observatory); heavy water and heavy water with salt for more intricate neutrino observations (Sudbury Neutrino Observatory)-allowing for the potential solution of the solar neutrino problem (Ahmad 2001); germanium, silicon, sodium-iodide, xenon and calcium tungstate in the attempts to detect dark matter (DAMA, CDMS, HDMS, UKDMC, CRESST); and finally, the various phases of different liquids and gases used in particle detectors, such as the cloud, bubble and wire chambers. Since all these interactions additionally depend on the existence and understanding of various subatomic particles, such as protons, electrons, and the $\mathrm{W}$ and $\mathrm{Z}$ bosons, of various forms of radiation such as gamma, 
beta and Cerenkov, and, lately, of computer technology, we can say that these observations actually employ very sophisticated interaction mediums involving many layers of matter, radiation and electronic circuitry. And as new forms of matter and radiation are discovered, they, in turn, can be used to detect newer forms.

We can also come to know the world through our touching, smelling and hearing. What sort of world does the blind person "see"? He uses his remaining senses to form images of the world around him. How do these images compare with the ones that we see with our eyes? And what more could he "see" if these other senses were enhanced by technology? To take the analogy further, imagine a planet where life evolved differently. Say the organisms there possess sensory organs radically different from ours. How does their world appear to them? How do they perceive the sun and stars? The "same" universe we live in: how does it look to them? It is not necessary that perception requires light or, even, electromagnetic radiation. Many forms of physical interaction can be used, and the form used cannot but effect the perceived observation.

2.4.4. Range. The last component of an observation we will consider is range. We can explain this best by giving some examples. Consider the motion of the planets in the gravitational field of the sun. By confining our measurements to the outer planets, we get an observation of gravitational phenomena that fits Newton's theory. By extending our measurement to planets closer to the sun where the gravitational field is stronger, we start to find differences, for example, the precession of Mercury's orbit. This novel observation lend support to Einstein's theory of gravity, displacing Newton's theory. Thus by observing gravitational effects at different field strengths, distances, or masses, or in other words, different ranges, we may find anomalies. Of course, by increasing the precision of the measurement in the original range, for instance, by observing the motion of the outer planets more precisely, we may improve our data and come to the same conclusion. Changing the range, however, will sometimes yield a result that is more obvious, though it may be more difficult to experimentally accomplish.

Although the Mercury measurement involves stronger gravitational fields than the outer planets, it is still considered a relatively weak field. For example, if $g$ is the gravitational field on the surface of the earth, then the sun's gravitational field within the solar system ranges from $7 \mathrm{~g}$ (sun's surface) to $0.004 \mathrm{~g}$ (Mercury) to $4 \times 10^{-7} \mathrm{~g}$ (Pluto); by contrast, the field near a neutron star or black hole is on the order of $10^{8}$ to $10^{11} \mathrm{~g}$ or greater. The limited observations of strong field effects currently support general relativity, though more observations are being planned in the future (Will 2001).

Conversely, we can ask how does gravity behave in extremely weak fields. By observing the orbits of stars at the distant perimeter of galaxies (Babock 1939; Rubin 1970) and the relative orbits and motions of galaxy clusters (Zwicky 1933; Smith 1936), where the fields are around $10^{-11} \mathrm{~g}$ or smaller, astronomers found that the motion is indicative of much more mass than indicated by the luminosity of the galaxies. Although, in a Quine-Duhem fashion, they have proposed new theoretical elements to account for these anomalies, such as the popular but yet undetected dark matter, others are toying with alternative gravitational theories that behave differently in extremely weak fields, the purported MOND (Modified Newtonian Dynamics) theories (Milgrom 1983a, 2003; Soussa 2003).

Independently of the field strength, we can ask how does gravity behave at different distances. For example, though it has been difficult to conduct gravitational experiments at the sub-millimeter scale (see Long 1999 and Adelberger 2003 for a summary), many physicists speculate that quantum gravity and/or string theory effects may come into play (Arkani-Hamed 1998; Adelberger 2003). The remarkable Eot-Wash experiments (Hoyle 2001) has managed to test the inverse square law down to 0.2 millimeters, when once the limit seemed to be more than a few millimeters. Though no deviation from theory has been found, the Eot-Wash team plans to improve to less than 0.1 millimeter. Elsewhere, Amelino-Camelia $(1998,1999)$ proposes testing quantum gravitational effects by making highly precise observations of astrophysical phenomena using space based gamma-ray and gravitational 
wave observatories. In one bit of exciting news, Nesvizhevsky (2002) and colleagues have preliminarily observed neutrons occupying discrete quantum states within the earth's gravitational field, at heights on the order of micrometers.

At the large scale, the previously mentioned anomalous observations of stars and galaxies in a weak field may perhaps be due to the great distances involved, $10^{9}$ to $10^{11} \mathrm{AU}$ (where 1 $\mathrm{AU}$ is the distance from the sun to the earth, and $40 \mathrm{AU}$ from the sun to Pluto). And at larger scales, recent observations of the velocities of distant ( $\left.10^{14} \mathrm{AU}\right)$ supernova (Perlmutter 1998) and of thousands of galaxies spread out over $10^{13} \mathrm{AU}$ (Zehavi 1999) tentatively indicate that the expansion of the universe is surprisingly accelerating. Again, some theorists propose new theoretical elements, such as dark energy or quintessence, while others propose modifications to general relativity (Deffayet 2002; Moffat 2004; Caldwell 2004). (For further references on large scale gravitational experiments see Adelberger 2003.)

We briefly give some additional examples of novel observations (or the possibility thereof) via range change, which may be more thoroughly followed up in the accompanying citations. Like gravity, the other fundamental interactions can also be measured at different ranges. For example, although QED is considered a highly accurate theory of the electromagnetic interaction, it has yet to be precisely scrutinized under strong electrical fields. For a survey of current and future strong field electromagnetic tests, including a strong field test of the electron's AMM and binding energies (QED Lamb shift), see Anderson (2001). Similarly, although quantum physics is considered a highly accurate theory, it has yet to be tested over large scales of distance, time, mass, etc. (see Leggett 2002 for a survey). In the field of particle physics, experimentalists are always trying to build higher energy particle colliders so that they can look for new particles and clues on unification. Current colliders are already producing novel particles that challenge the Standard Model (Choi 2003; Evdokimov 2004). The Large Hadron Collider at CERN, to come on line in 2007, will reach energy levels of $14,000 \mathrm{GeV}$, the highest yet, but still a vast distance from the Planck energy of $10^{19} \mathrm{GeV}$, where it is believed quantum mechanics and gravity merge. What novel discoveries await us on this long road, and then thereafter? As another example, consider the difference in behavior of rods and clocks at small and large velocities. By observing matter at sufficiently large velocity ranges, we find that clocks run slower and objects contract. This difference supports the special theory of relativity over Newtonian mechanics. Other examples include an observation of a decrease in entropy in smaller systems over shorter time scales (Wang 2002) - a recently predicted violation of the second law of thermodynamics (Evans 1993); a reanalysis of the star formation time line by observing lighter mass galaxies (Heavens 2004); and anomalous observations of the cosmic microwave background radiation at larger spatial angular separations (Bennett 2003; Contaldi 2003), possibly supporting different geometric models of the universe (Efstathiou 2003; Luminet 2003). Generally speaking, for any physically observable quantity, such as mass, momentum, energy, distance, temperature, gravitational force, electromagnetic force, etc., we can conduct our experiments in ever wider ranges as time and technology advance. By expanding the range it is possible to get results that were not evident or obvious in previously observed ranges.

Precision, perspective, interaction and range: we have identified four ways in which an observation can vary. By improving or modifying these components, we can have novel and, perhaps, anomalous experiences of nature. This meta-empirical evidence is of principal importance because it allows us to equate the notion of incompleteness in scientific theories with the notion of incompleteness in natural experience. We could not have one with out the other. This identity emphasizes the intimate connection between theory and experience and, at the same time, exposes the paramount significance of their inseparability.

2.5. Quantification of the Philosophy: an Information Metaphor. We can now paint a clearer picture of the role theory and experience play in science. The interaction between nature (or the "outside world") and our sensorial-cognitive system results in the production of a flux of 
information..$^{21}$ Our understanding of this flux as experiences and, on a more broader scale, as theories which integrate many different experiences is an attempt at finding patterns within the flux. In other words, science can be viewed as a problem of pattern recognition within a given information space of sensorial-cognitive flux. The incompleteness principle implies that such patterns cannot fully represent all the information within this space. This is because the space has an infinite amount of detail. ${ }^{22}$ At a specified level of detail (e.g., observational precision), we may be able to find a pattern that most closely approximates the information. But if we zoom in to a higher level of detail (e.g., higher observational precision), there is no guarantee that the found pattern will hold. Each higher level of detail arises in a nonalgorithmic and non-predictable fashion. We will further clarify this picture in Part III, where we consider the mathematical dimension of incompleteness.

2.5.1. Science as Pattern Recognition. We can make an analogy here using digital images. Imagine we take a digital photograph of some natural scenery. We can shoot the scene at various pixel resolutions. Someone looking at a low resolution image of the scene, who has not seen the scene with her naked eye, may perhaps interpret the image incorrectly. She may identify certain patterns of pixels that may not exist at a higher resolution. Moreover, the image will appear to represent many possible scenes. The lower the image resolution, the more erroneous of a guess is she likely to make and the more guesses is she likely to entertain. As the resolution of the image approaches closer to that of the human eye, she is able to narrow the possibilities and better identify the correct scene. In actuality, stepping back away from the analogy, none of us have witnessed the "real" scene of natural experience. We are always looking at a resolution that is infinitely far below the "real" scene. We must take our best guess based on this resolution until technology allows us to look at the next higher resolution. Recall the example above where the data on planetary motion was described using mathematical models. At each higher level of detail in the data, we found novel models arising in a non-predictable fashion.

Let us make this explanation a little more precise. Consider an image which is being viewed at its full resolution, i.e., we can know the color of every pixel in the image. To streamline our argument, let us simplify the image to black and white, and consider the representation of geometric patterns instead of natural scenery (see diagram). Even though we are now viewing a full resolution image, we may still give multiple correct descriptions of the image. We may say, for example, that it is a square with two diagonals drawn from pairs of opposite corners, or two isosceles triangles, each perpendicularly bisected, or four smaller isosceles triangles. All the descriptions, though in a certain sense being different, can be considered to be mathematically equivalent (isomorphic).

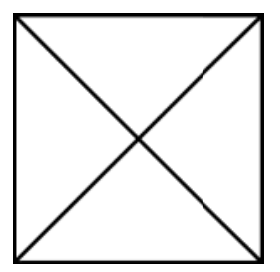

Another way to see this is to write out the image as a binary number. For example, say our image turns out to be the binary number 1110 . Translating into decimal notation, we get 15. We can express 15 in many ways: $3 \times 5,10+5,17-2$, etc. All these different expressions imply the same number. What we are getting at is that, given a full resolution image in which

\footnotetext{
${ }^{21}$ Alternatively, we may say the interaction between sensorial and cognitive processes. For our purposes, it does not matter where we draw the putative line in the physical interaction between nature and ourselves.

${ }^{22}$ Though it may be difficult to appreciate, mathematicians have learned that infinity comes in many sizes and that they do not encompass all there is in the mathematical world of objects. At present we are unable to identify the size of the infinity that we are dealing with; therefore, we will say it is at least the smallest infinity, $\aleph_{0}$, the size of the set of all natural numbers. (Though there is some reason to believe that it may be at least the next largest infinity, $\aleph_{1}$, the size of the set of all real numbers, a point to be explicated at a later date.)
} 
all the colors of the pixels are known, then there is only one isomorphic class of descriptions for that image. If, on the other hand, we are uncertain about the color of certain pixels-the ones that only come into view at higher resolutions-then there will be more than one isomorphic class of descriptions. For example, after translating an image into binary, we get 11?01?10, where "?" represents our ignorance of the pixel value, then this image has the potential to represent four different ones, corresponding to the four different binary numbers that could result after filling in for the missing question marks.

If we now interpret the images as empirical data and the descriptions of the images as scientific theories, we get the following theorem:

UDT Requirement Theorem. The number of isomorphically different theories on a given domain of phenomena is proportional to the amount of incomplete empirical data on that domain. Specifically, if the empirical data on that domain could be complete, then there would be only one correct isomorphic class of scientific theories for that domain. Moreover, all the theories in this class could be complete. ${ }^{23}$

This theorem is simply a more explicit rendition of the implication between the underdetermination of theory (UDT) and the novel experience problem; it defines the applicability of UDT. The latter asserts the existence of non-isomorphic theories for a given domain of phenomena. By the above theorem, this is only possible if the empirical data is not completely known. This insight clarifies a puzzling aspect of UDT: how two or more mutually inconsistent theories can possibly describe the same empirical data (for example, see Bird 2003; Sankey 1997). It is only so because the data itself is inherently fuzzy, just as a low resolution image appears to represent distinctly different possibilities. The inherent incompleteness of empirical data allows for the existence of multiple theories.

One may wonder why we have put forth a theorem in which the incompleteness of empirical data can vary or even be zero, after earlier asserting that empirical data is infinitely incomplete. We do stand by our earlier assertion, but this theorem helps us to gain a clearer understanding of the relation between incompleteness and underdetermination. It also explains why at times scientists are willing to entertain multiple theories, and at other times not, even assuming that all the technical problems have been resolved in these theories. Although the principle of experiential incompleteness asserts that empirical data is infinitely incomplete, the amount of perceived incompleteness can vary in time. One reason for this variation is the cyclic behavior of scientific research as elucidated by Kuhn (1970). During revolutionary periods, it appears that there are holes in our empirical knowledge and that we are continually trying to fill them with discoveries, whereas during the stable periods, there is mostly supporting data being gathered. If scientists believe their empirical data is hardly lacking, then any claims of underdetermination would only fall on deaf ears; whereas, if the empirical knowledge of some domain is scant, they are willing to entertain many a possibility. The lesser the empirical knowledge, the greater the extent of underdetermination.

We can produce a similar theorem for the underdetermination of observation (UDO). Though first we need to define the notion of "isomorphically different observations on a given domain of phenomena." We treat the totality of all observations on a given domain as a singular observation. For example, if there were ten different observables on a given domain, then each set of possible outcomes for all ten observables represents a singular observation and one that is different from another set. By isomorphically different, we mean that no two sets can be reduced to the same set; they are mutually incompatible (see the explanation a few paragraphs back regarding isomorphic different descriptions of digital images).

\footnotetext{
${ }^{23}$ At this time, we are unable to offer a more precise relation of the proportionality. This is due to a lack of mathematically formal definitions for the concepts of empirical data and scientific theory. For example, by information theory, it may appear at first blush that the number of isomorphically different theories is proportional to $n^{2}$, where $n$ is the number of incomplete bits of empirical data. However, the necessity to incorporate theoretical language when describing empirical data may preclude all the different n-bit combinations from only specifying purely data, and thus the proportionality may be less than $n^{2}$.

An example of isomorphically equivalent theories on the same empirical domain are the Schrödinger (wave) and Heisenberg (matrix) formulations of quantum mechanics.
} 
UDO Requirement Theorem. The number of isomorphically different (mutually incompatible) observations that a theory on a given domain of phenomena can accommodate is proportional to the amount of the theory's incompleteness. Specifically, if the theory could be complete, then there would be only one correct isomorphic class of observations that can be accommodated. Moreover, all the observations in this class could be complete.

This theorem more clearly quantifies the idea expressed in Section 2.2.3, concerning the relation between UDO and theoretical incompleteness. There, we argued that UDO critically depends on theoretical incompleteness. This theorem quantifies that dependence. Also, just as in the case of experiential incompleteness, theoretical incompleteness is infinite, and therefore, as asserted by the Quine-Duhem thesis, a theory can accommodate any amount of seemingly incompatible observations (but see Section 3.4.3); however, similarly to experiential incompleteness, the perceived amount of theoretical incompleteness can vary too.

The gist of the UDT and UDO requirement theorems is that the extent of theoretical underdetermination by observations and the flexibility of theories in accommodating novel observations is dependent on the extent of observational and theoretical incompleteness, respectively. UDT, UDO, and the Quine-Duhem thesis as a whole hold no epistemological weight without observational or theoretical incompleteness.

2.5.2. Science as Data Compression. The science as information metaphor has more to teach us. Following the lead of Solomonoff (1964) and Chaitin (1970, 48-49), we now consider the idea of science as data compression. By recognizing patterns, such as a circle or ellipse, within a digital image, we are finding a compact way to express what would otherwise be just a long description of the colors and positions of each pixel. Similarly, by recognizing patterns in long strings of binary numbers, such as $010101010 \ldots$. . we can give more condense descriptions. Compression has become a measure of recognition or description quality: the best descriptions are the smallest ones.

In science, then, experiential and theoretical descriptions are attempts to compress the vast amount of information arising in our sensorial-cognitive system, again the best descriptions being the smallest. For example, it is far more concise to give the initial position and velocity of a planet and Newton's laws than to give an arbitrary large number of position and velocity values themselves. Similarly, it is far more concise to give the theory of the atom than to describe the behavior of the many chemical elements individually. All descriptions in science, from geometric models to equations of force, and from sub-atomic structures to biological structures, serve to compress large amounts of observational data into smaller, finite patterns. The adoption of computers in theoretical modeling further supports this metaphor: theories become programs and their outputs become observational predictions.

The data compression analogy allows us to reframe the question of what is the best theory on a given domain of empirical phenomena as what is the smallest compression that can be achieved on the corresponding empirical data set. Or, in other words, what is the absolute informational content of the empirical data set? By the novel experience problem, it is not possible to determine this. For assume we find some smallest compression algorithm (theory) to describe a series of binary digits (some finite data set). For example, if the binary digits are 001011001011, then a theory would be repeat 001011 two times. But upon probing nature deeper, we find that the data set has grown to 01010101010101010101 . Even though the data set is larger, we can find a shorter description: repeat 01 ten times. Unification examples from the history of science aptly illustrate this point. The informational content of Maxwell's electromagnetic theory is much less than the sum of its disparate predecessors: the electric, magnetic, and light theories. The same can be said of Einstein's theory of gravity versus Newton's disparate theories of gravity and motion. In these cases, further details have emerged in our empirical investigations that allow us to simplify our descriptions, but it is 
not necessary that simplification is the rule. Generally, as the empirical data grows, it is not possible to determine the size of the resulting theoretical models.

The above result can also be expressed in terms of communication theory. We can view science as trying to accurately communicate our experiences of nature. Incompleteness then implies that there is no ideal communication code in which to convey these experiences. Now, it is a well known theorem in communication theory that there is no absolute measure of information. Information can only be measured relative to some model. Depending on the model, the information content of a string of " $n$ bits" can have a size from zero bits to any arbitrary number of bits larger than $n .^{24}$ Similarly, in science we choose (explicitly or implicitly) some model, interpretation, or ambient theory in order to communicate our "raw experiences." This point has been recognized in Suppes (1962) and McAllister's $(1997,2003)$ idea of data models and data patterns, respectively. The insight to be gained here is that empirical data comes in multiple interpretations only because it is incomplete, the point made by the UDT requirement theorem.

2.5.3. The Entropy of Physical Systems. As with the information measure of empirical data, the novel experience problem also implies that the entropy of physical systems is not an absolute quantity either, a point recognized by Weintstein (2003). Although Wienstein does not explicitly state the novel experience problem or theoretical incompleteness, he argues that the entropy of a physical system depends on the description of the system, of which there can be no fundamental one:

In conclusion, then, the entropy depends not only on the substance, but on the description of the substance, and there is no most-fundamental description. Which description is appropriate depends on which variables one is able to control, or interested in controlling. As is the case with algorithmic complexity and relative velocity, entropy can be contextualized, and entropy relative to a description can be considered an objective property of a physical system. But there is no most-fundamental description, just as there is no most-fundamental Turing machine or inertial reference frame. (1252, italics in original)

Again, as with the notion of data models, Weinstein's notion of contextualized entropy is possible because the measurements on a physical system cannot be complete. If they could be complete, then the entropy would be absolute. Indeed, Weinstein recognizes this point:

But the problem is that one can introduce further state variables as well, not only by discovering new sorts of properties, but by manipulating the same properties in finer detail... This process is limited only by the fact that, at extremely small scales, the system ceases to display equilibrium behavior. (1252, italics in original)

It is obvious from this passage that Weinstein implicitly assumes the novel experience problem to hold. Though, it is unclear whether he goes so far as to subscribe to the principle of theoretical incompleteness.

Also, as Weinstein points out above, it is unclear whether the notion of entropy continues to hold at extremely small scales due to thermal or quantum fluctuations. We further note that the Bekenstein bound $(1974,1981)$, which limits the maximum allowable entropy per given volume of space, may conflict with the notion that the entropy of a system can be arbitrarily large. Presently, it is unclear how to resolve these potential issues. Perhaps a new understanding of entropy and information, say, founded on quantum informational approaches, may help (Zeilinger 1998, 1999; von Baeyer 2003).

\footnotetext{
${ }^{24}$ This result, like its scientific counterpart, is only possible because the information potential is infinite. If, in communication theory, we limit our message source to some finite size, then it is possible to put a cap on the information size of any particular message, even though we are unable to determine the exact size of the message. To do the latter, we need to, of course, pick some preferred model.
} 
2.5.4. Can a Computer Simulation Ever Represent Reality? By extending the digital images analogy we can examine an interesting question in ontology. For consider, instead of a 2D image, a 3D virtual reality (VR) computer simulation of our experience. This is identical in principle to a two dimensional digital photograph, only we are expanding the visual matrix into the full three dimensions (like a hologram), and adding digital "photographs" of the remaining human senses: hearing, touch, smell, and taste. The addition of physical (causal) laws into the program allow us to add the dimension of time into the VR world, making it dynamic instead of static.

If we were immersed into such a virtual reality world without knowing it, we may be hard pressed to determine if the world is real or a simulation (a phony). The better the simulation, the harder it will be to make that determination. On the one hand, someone (a prankster) is trying to simulate observations, and on the other, we are trying to determine whether they are valid.

This raises the classic question of whether this world, which we are all commonly experiencing, is a computer simulation or real (natural). Theoretically, there is a way to determine this. By the novel experience problem, we can interact with the real world on an ever deeper level, and thus always improve the resolution of our experiences. In any computer simulation, we will eventually hit a brick wall, or limit of interaction, because there is only a finite amount of data that was programmed into the simulation. That is to say we will realize the simulation and probe past it into the real world. This contrast highlights a critical point we recently raised: the natural world (as experienced through our sensorialcognitive system) is infinite, while representations of it-theories, descriptions, computer simulations, etc.- -are finite. It also gives us another angle on the incompleteness of scientific theories: theories are incomplete because they are finite, while nature is infinite.

This finiteness explains why we may have a hard time to determine valid observations. At any point in time, all our descriptions of observations are finite, however large or small, and thus the observations can be mimicked, given enough artistry or technology. Though, as we said, the real world is infinite, so if we probe the simulation deeply enough, we should reveal the illusion. But keep in mind, that at this point, we are also probing past the limits of our actual knowledge of the observation, and that if we were probing the real observation, we would be collecting new data and thus adding to our original description. If we interact with the phony observation within the finite range of its original description, i.e., in a normal, every day capacity, we will not notice that it is phony. Moreover, recalling the theoretical caveat above, the description, though being finite, may be arbitrary large, and thus we may have to probe the observation for an arbitrary long time to determine whether it is real or a simulation. Therefore, while we may be able to determine whether simulations that we, ourselves, create are phony-because we know the limits of our own knowledge and thus how far to probe-we may not be able to do so if an outside agent whose knowledge is greater than ours creates them. Thus to answer the question titling this sub-section, reality cannot be (entirely) a computer simulation, but we cannot prove that our reality so far experienced is not a simulation.

Some critics may counter that there are finite mathematical representations that, nonetheless, have an infinite extension or realization and thus may be used to describe the infinite depth of nature. Such examples may be fractals, cellular automata, or even numbers like pi and the square root of two. Let us consider cellular automata, as they are perhaps a more stronger example in favor of such arguments. Cellular automata are given by finite rules, yet calculating according to these rules, we can get an infinitude of unpredictable patterns. It seems that since we have an infinitude of patterns, we may be able to use such mathematical entities to give a complete description for certain physical theories, or provide an uninterrupted depth of observer interaction in a VR simulation. This is a subtle point. In mathematics, one learns that infinity comes in many sizes and does not encompass all there is. In spite of these mathematical entities being able to generate an infinitude of patterns, there are certain patterns that they cannot generate. More precisely, given a complex enough 
cellular automata, it will be undecidable whether certain patterns appear or not (Wolfram 2002, 755). (This point will be examined in depth in Part III.) This undecidability will manifest itself as a limit of observer interaction in a VR program that employs cellular automata type rules. The undecidability can be resolved by expanding the rules of the cellular automata (expanding the size of the VR program), but then there will be yet another undecidable pattern. In this way, the cellular automata is essentially incomplete in addressing the world of its patterns. So while a cellular automata may provide an infinite amount of description or observer interaction for a physical theory or VR program, respectively, it cannot provide a complete or unlimited one, respectively. The bottom line is that all computer simulations, being necessarily given by a finite program, will have a limit of observer interaction.

\section{PART III: MATHEMATICAL CONNECTION}

3.1. Introduction. Our brief examination of cellular automata above glosses over some of the finer points of a milestone in mathematics: Gödel's Incompleteness Theorem (1931). By examining these finer points, we can place our philosophical theory within the more familiar context of mathematical incompleteness and undecidability, and give it a formal dimension that has thus far been lacking. We will keep the discussion to a minimal technical level.

Gödel's incompleteness theorem, in actuality, encompasses both his first and second incompleteness theorems. The first incompleteness theorem addresses the question of completeness, while the second incompleteness theorem addresses the question of consistency. Both questions and theorems are very closely related. Here we are concerned primarily with the question of completeness and, thus, primarily with the first incompleteness theorem. Henceforth, when referring to Gödel's incompleteness theorem, we are in fact referring to his first incompleteness theorem, unless stated otherwise.

Gödel's incompleteness theorem proved that the arithmetic of natural numbers-the addition and multiplication of zero and the positive whole numbers-cannot be given a finite description. More specifically, no matter how sophisticated a system of axioms one comes up with, as long as it is given as a finite (and consistent) system, there are statements of arithmetic (a.k.a. Gödel statements) that are neither provable or disprovable by the system and, hence, are undecidable. Immediately, we see an analogy between Gödel's theorem and the idea of incompleteness in scientific theories. This analogy is not a coincidence, but represents a strong connection between these two ideas. In what follows we will argue that the mechanisms which allow for scientific incompleteness finds a natural framework in the incompleteness theorems of mathematics.

3.2. Gödel's Incompleteness Theorem and Computational Irreducibility. To begin, we need to find out why is it that such a seemingly simple system as the arithmetic of natural numbers cannot be finitely described. Since its initial publication, Gödel's theorem has sporadically sparked further work in mathematical and related fields that has helped improve our understanding of the phenomena of incompleteness. For instance, it is now known that there are other systems that exhibit similar incompleteness properties. Shortly after Gödel, Turing (1936) showed that it is not possible to write a computer program that can decide whether all programs will ever halt or not (stop and produce some output or continue running forever). There will always be computer programs whose halting status is undecidable. Another example, due to Wolfram (2002), includes certain class of cellular automata, as was mentioned at the end of Part II. Chaitin $(1971,1975)$ has shown, via his algorithmic information theory, that given any algorithm or computer program, there exist numbers for which it is undecidable whether they are random or can be reduced to some pattern. Similarly, he has shown that the complexity of computer programs is an undecidable issue $(1974,2000)$.

From the study of these additional phenomena, researchers have been able to glean a common principle. We can state it briefly by echoing the words of Wolfram $(2002,788)$ : there 
exists certain infinite processes that are computationally irreducible. Such processes occur in arithmetic, cellular automata and computers, among other systems. As a simple example, consider a finite two dimensional black and white matrix. Some matrices can be described without specifying the color of every cell in the matrix, such as a checkerboard pattern. Though if the matrix is completely random, then we have no choice but to specify the color of every cell. In the latter case we say that the matrix has no pattern or is computationally irreducible. Now imagine that the matrix has an infinite number of cells. Like in the finite case, there exist infinite patterns-patterns that as you keep zooming in, repeat identically or in some sort of algorithmic or recursive fashion. If, however, the matrix is infinite and random (more precisely if it has an infinite random portion, an infinite number of random finite portions, or an infinite number of patterned finite portions that vary randomly), then no amount of finite information is sufficient to describe it. In such a case we have non-finite computational irreducibility. Continuing this analogy, we can say that mathematicians knew they were dealing with an infinite matrix because the natural numbers go on forever, but it was not until Gödel that they realized that the infinite matrix could not be reduced to some finite set of patterns (finite set of axioms).

As a more robust example of computational irreducibility, let us consider a direct example from the files of arithmetic. Specifically, we consider a class of arithmetical equations known as diophantine equations. (For the most part, these are just normal polynomial equations set equal to zero.) The equivalent of Gödel's theorem here is that there exist diophantine equations for which it will be undecidable whether they have solutions or not. How is this possible?

Let us think about what it means to say that it is undecidable whether or not a particular equation has a solution. Undecidability implies that it is not possible within the given system of arithmetic to prove that the equation has a solution or prove that it does not. This further implies that the equation in fact has no solution, but that we cannot prove this in the given system. (For if it did have a solution, we would eventually be able to prove it.) To decipher the mystery we must focus our attention on why we cannot prove that it does not have a solution. To this end, consider equations that do not have solutions and for which we can prove it, say $2 x-7=0$. No matter how many natural numbers we try, we will not find a solution to this equation; moreover, we can prove it. In attempting to solve it, we can simplify the equation to $2 x=7$. Seven is odd and therefore not divisible by two. Thus we can prove that the equation has no solution. Not all such proofs of non-solvability are this simple. Consider the famous case of Fermat's last theorem, regarding the equation $x^{n}+y^{n}-z^{n}=0$. Alas, Wiles (1995), building upon the work of many others, proved that any variation of the equation where $n$ can be greater than two has no non-zero solution for the variables $x, y$ and $z$. The proof was immensely long, taking hundreds of pages.

Both the above proofs are making assertions about the natural numbers: that no (nonzero) numbers from that infinite set of numbers form a solution to the respective equations. These proofs or theorems of non-solvability serve to compress some particular information about the infinite set of natural numbers into a finite form. Theorems of non-solvability have no choice but to do this, as they have to address all the infinite natural numbers, as all are candidates for a solution. The fact that we cannot find a non-solvability proof for the undecidable diophantine equation means that we are unable to find any such finite compression. The equation in question corresponds to some infinite process within the arithmetic of natural numbers which is not finitely compressible using the rules of the given arithmetical system. We can expand the rules to help us decide on this equation, but then Gödel's theorem tells us that there will always be another undecidable diophantine equation. In fact, there are an infinite number of undecidable diophantine equations. We therefore see that the ultimate cause of Gödel incompleteness is that there exist infinite processes within the arithmetic of natural numbers that are simply not reducible to a finite description, no matter how large. This is the same reason why undecidable phenomena occur within cellular automata and computer programs. Any rules or descriptions we come up with to explain any of these systems will always be finite, but as there are computationally irreducible infinite 
processes going on within these systems, our finite descriptions of them will forever remain incomplete.

Chaitin's algorithmic information theory provides another take on the problem. Any theory or description contains a finite amount of information as is determined by its axioms. If put to the theory a question which contains significantly more information than the theory-which is possible in systems that contain a computationally irreducible amount of information - then it will be undecidable what the answer will be (Chaitin 1974; Calude 2005). The only way to resolve the undecidability is to enlarge the axiom base and, hence, the information content of the theory. In this vein we can say, for instance, that undecidable diophantine equations contain significantly more information than the axioms of the given arithmetical theory, or that computer programs whose halting status is undecidable contain significantly more information than the given computer program that was written to decide whether programs halt or not.

Gödel's incompleteness theorem, or its modern form, computational irreducibility, provides us with a natural mathematical framework for scientific incompleteness. We suggest that the interaction between nature and our sensorial-cognitive system involve computationally irreducible infinite processes that prevent us from achieving complete descriptions of any sentiently experienced phenomena. This fits in with the picture we painted in Section 2.5 of science as a problem of pattern recognition within a given information space of sensorial-cognitive flux. As this space contains a irreducibly infinite amount of information, no amount of finite patterns can serve to completely describe it. This is also why mathematical models or computer programs, all of which are finite, cannot be used to provide a complete scientific theory or virtual reality simulation, respectively.

At this point we must pause and say that we do not yet know how computationally irreducible processes could arise within the sensorial-cognitive system. What we want to recognize here is the plausible case for the existence of such processes and what their consequences are for scientific understanding. Nonetheless, we suspect that if they do exist, then they may be connected with a fundamental inseparability in the interaction between nature and our sensorial-cognitive system, which we postulated in Section 2.1 manifests as an inseparability between sensorial and cognitive processes. Regardless, we emphasize our prior contention that scientific incompleteness, along with any of its associated mechanisms and properties, such as possibly computational irreducibility, are not purely external characteristics of nature, but inherently arise out of our sensorial-cognitive system and its interaction with nature.

3.3. Requirements for Incomplete Systems. Let us continue our mathematical examination of scientific incompleteness and see how additional insights on the phenomena of mathematical incompleteness help us to better understand the scientific counterpart. A central question is what is it about all these different systems that allow them to be incomplete and what is it that distinguishes them from other systems that are complete? It is clear from the previous discussion that incomplete systems are computationally irreducible, so perhaps it is more accurate to ask what gives rise to the latter. Both Wolfram (2002) and Hofstadter (1979), among others, have addressed this issue. While it is a matter of continued research, we can tentatively identify two main causes: (1) infinity and (2) a minimal level of complexity in the rules or axioms of the system.

3.3.1. Infinity. The first cause is almost obvious. Any finite system can be finitely described, even if there is no order or pattern to it-though the description may be quite lengthy. Consider the system of arithmetic. If we limit the natural numbers to a maximum, say one million, then we can prove whether all diophantine equations have a solution or not: we try all numbers from one to a million and see if we find a solution. Similarly, for computer programs, if we limit the memory of the computer to a maximum size, we can determine the halting status of all programs requiring memory up to that maximum size. Wolfram (2002, 255-260) has shown that no matter how sophisticated a cellular automata one has, if the 
width of the pattern is bounded to a maximum size, the automata will eventually repeat itself as it evolves along its length.

3.3.2. Minimal Complexity. But infinity is not sufficient to guarantee incompleteness. For instance, it has been proven that the arithmetic of natural numbers which excludes either addition (Skolem arithmetic) or multiplication (Presburger arithmetic) is complete (Skolem 1930; Presburger [1929] 1991). Wolfram (2002, 51-58, 231-235) has demonstrated that unbounded cellular automata with simple rules exhibit repeatable or algorithmically predictable behavior. In order for incompleteness to exist, the system should also have a certain level of complexity. For arithmetic, we can identify what is involved in achieving this level: it should be able to represent what is known in mathematical terms as all the general recursive functions. ${ }^{25}$ This is sometimes referred to as an expressiveness condition. In simpler terms this amounts to the ability to add and multiply two numbers, and also compare two numbers to see if they are equal or whether one is larger or smaller than the other. For computers, it involves the requirements as specified by Turing (1936) or, equivalently, the capability to perform the Boolean-logical operations of AND, OR and NOT, among other functions. A computer that meets these (and the infinite memory) requirements is called a (universal) Turing machine. Wolfram has thus far been unable to identify what exactly is needed for cellular automata to achieve incompleteness, though he has been able to identify certain characteristic properties. Moreover, it is presently unclear what is the common mechanism that underlies the complexity of all these different systems.

There is strong reason to believe that there is such a common mechanism. For we know that some of these complex systems can, at least partially, simulate other such systems. A modern day example is computers representing other computers by means of operating system emulation software. Going further, the Church-Turing thesis stands unrefuted in its assertion that all functions or mechanical decision procedures (algorithms) which can be respectively computed or carried out (on some device) can be done so on any computer meeting Turing's minimum requirements, given enough memory and time (Church 1936; Turing 1936). All such computers are equivalent in their computational capability (although their memory and speed may vary). The MRDP theorem of recursion theory, which establishes the equivalence of certain computable relations (recursively enumerable relations) to diophantine relations (based on diophantine equations), extends this generalization to the language of arithmetic (Matiyasevich 1970). Since all computer program inputs and corresponding outputs can be viewed as natural numbers (in binary form), it is easy to see that all programs can be represented as an arithmetical function which maps one set of natural numbers onto another. As an actual example, Chaitin constructed a diophantine equation 900,000 characters long with 17,000 variables which corresponds to a general purpose LISP program (Chaitin 1990,17). Turning to other systems, in a remarkable series of examples, Wolfram $(2002,644-673)$ has demonstrated a variety of different systems simulating others, including cellular automata simulating other cellular automata and, even, computer and arithmetical operations. Armed with these examples, Wolfram claims that there is a certain sense of equivalency or universality in all computational systems that meet a minimal level of complexity. Whether this is the case or not, it is generally accepted that many a formal system with symbols and rules for the manipulation of those symbols can be represented on a Turing machine or within the arithmetic of natural numbers. This will turn out to have implications in our upcoming considerations on self-reference in formal systems.

3.3.3. Infinity and Complexity in the Human System. So while we cannot identify the common mechanism of complexity, there is tentative reason to believe that infinity and complexity play a role in incomplete systems. If so, then they may also be involved in the processes of human experience and thought. From our discussion of the novel experience problem, we can see how infinity plays a role in experience: we can always improve the

\footnotetext{
${ }^{25}$ Technically, unless arithmetic is infinite, it cannot represent all the general recursive functions. Thus some might argue that the infinity requirement is part of the complexity requirement.
} 
precision of our experiments. Similarly, for our theories, we can always ask what is behind any theoretical construct. Thus there is an unboundedness in our ability to probe both our experiences and thoughts.

How complexity plays a role is more difficult to address, partly because we do not yet understand the common mechanism underlying all the different incomplete systems. We can best surmise that (1) there are sufficiently complex processes going on within the human (and perhaps animal) sensorial-cognitive system as it interacts with its environment and (2) that some capability equivalent to, say, the expressiveness condition of arithmetic must exist in any language strong enough to describe all our experiences. Less expressive languages may exist that can completely describe some aspects of our experience, but such a language will not be strong enough to describe all aspects of it, just like the Presburger or Skolem formalism (see Section 3.3.2) can describe some aspects of arithmetic completely, but cannot describe all aspects.

3.4. Arbitrariness in Theories. When in incomplete mathematical theories we encounter undecidable statements, it was earlier pointed out that the resolution lied in expanding the axiom base or information content of the theories. What we failed to mention is how we go about such an addition. Do we make the undecidable statement an axiom of the theory, or do we adopt its negation or some other variation? What algorithms or formal guidelines do we use to make such a decision? The simple answer is that since the statement is undecidable, there are no such guidelines; it is arbitrary. We can add the statement, its negation, or some other variation. The theory has nothing to say about the statement. It is the same way with the original axioms of the theory; the axioms are themselves undecidable statements. When we construct a theory, we start off by assuming a certain number of statements as true without proof, i.e., taken on faith, guts, intuition, or on the basis of some other nonformalizable notion. Another way to see this is in terms of Chaitin's algorithmic information theory. There randomness is defined in terms of incompressibility: something is random if it cannot be compressed into a shorter description (Chaitin 1970). The axioms of a theory serve to compress the larger information content of all its theorems into a smaller size (Chaitin 1975). In other words, we can find common patterns among all the different theorems. However, since we cannot compress the axioms, themselves, any further, we can find no common patterns among them; as Chaitin (1975) says, they are random.

3.4.1. The Role of Truth. Such arbitrariness in the expansion of a theory or in the selection of its axioms prompts one to question the role truth plays in theories. Before Gödel's theorem, the assumption was that there was some totality of mathematical truth awaiting to be finitely axiomatized. Gödel's theorem, of course, proved that such a finite axiomatization was not possible; however, in order to preserve the notion of truth, many have adopted a mindset that there is a permanent edifice of truth that, although could not be know entirely, could be revealed bit by bit. Such a Platonic outlook arose out of a misinterpretation of Gödel's theorem usually written thus: "Gödel's theorem states that there are true statements of arithmetic that are unprovable." Therefore, when we come across such statements, we should adopt them as axioms, for Gödel's theorem tells us they are true.

It is easy to see how such a misinterpretation may arise. Recalling the example of the undecidable diophantine equation (Section 3.2), we saw there that the diophantine equation in fact has no solution. Is it not therefore correct to adopt an arithmetical axiom saying that the equation has no solution? It is, but as Gödel's theorem tells us that the statement is undecidable within the original theory, its negation, instead, may be added to the original axioms to also create a consistent new theory. How is this possible? The confusion is due to our expectations and assumptions about what the theory describes conflicting with what the theory actually tells us. It is the Platonic idea that somehow we have intimate and direct access to the world of arithmetic (and mathematics generally), and that we are merely trying to find descriptions within language, via our theories, that match this world. If we assume arithmetic behaves one way, then there is no solution, but if we assume it behaves another 
way, there is a solution. There is no ideal world of arithmetic that favors one assumption over the other. By extending the axiom base of a theory, we are extending the world that the theory talks about. Thus when we add the above negation to the theory, we are now saying that there is something more to the world of arithmetic than we thought, and this something more will allow the negation to be true within the new theory. Whether any of these infinitely many undecidable diophantine equations (or Gödel statements) or their negations are of practical mathematical importance is matter of current research. For an in depth account of some of these points, see Hofstadter (1979, 451-456).

The above is not merely an abstract idea nor novel to Gödel's theorem. We can find many examples in mathematics of undecidable statements that, although are not examples of Gödel undecidability, leave open the way for multiple versions of a theory. As a classic example, consider the fifth axiom of Euclid's geometry. This is the standard geometry we all were taught in school. As this is an axiom, it is undecidable within the theory comprised of Euclid's first four axioms. Therefore we should be able to create variations on Euclid's original geometry by adopting different versions of the fifth axiom. Indeed such variations exist-spherical geometry, hyperbolic (Lobachevsky-Bolyai-Gauss) geometry, elliptic (Riemannian) geometry-and have practical applications on various curved surfaces or spaces, as in Einstein's theory of gravity. An example from set theory is the generalized continuum hypothesis. For a long time it was unclear whether the hypothesis or its negation could be proved from within the standard Zermelo-Fraenkel formulation of set theory (ZFC). Eventually it was proven by Gödel $(1940)$ and Cohen $(1963,1964)$ that the hypothesis was undecidable in the given set theory, thus bifurcating the theory. As another example from set theory, by negating what is known as the foundation axiom, mathematicians have been able to arrive at a different version of the theory that allows them to deal with sets that possess an infinitely nested structure (Forti 1983; Aczel 1988). It is not all the time that when we vary the axioms or other undecidable statements of a theory, we get a theory whose practicality or, even, comprehension is immediately obvious, but we can definitely say that it is a consistent theory about something or another.

From these examples and the previous considerations, we see that the notion of mathematical truth is a profoundly relative one. Statements are true relative to a world or, more specifically, a model that a theory aims to describe. And many mathematical theories, including arithmetic, are unable to uniquely determine their models, even up to isomorphism. Moreover, any statement, as long as it is not logically inconsistent relative to the axioms of a given theory, is true within the theory comprised of those axioms and the statement, for some model. Accordingly, a more appropriate interpretation of Gödel's theorem would be: "There are true statements relative to a given model (or world) of arithmetic that are unprovable." Still, an even more accurate (and simpler) interpretation would be: "There are statements relative to a given theory of arithmetic that are neither provable or disprovable." The salient point here is that given an undecidable statement, we can extend a theory in many directions, not just one (true) direction. Failure to recognize that Gödel's incompleteness theorem undermines the notion of an absolute, or singular, truth in mathematics would be a grave oversight. In Section 3.5.2, we will see that Tarski's undefinability theorem (1933), a close cousin of Gödel's theorem, demonstrates that no sufficiently expressive formal system can provide its own truth definition. ${ }^{26}$

3.4.2. Arbitrariness in Scientific Theories. The rise of arbitrariness and the decline of truth in mathematical theories brought on by the development of Gödel's incompleteness theorem finds a parallel in scientific theories. However, here it was the recognition of arbitrariness that came prior to that of incompleteness. This arbitrariness came in the form of the Quine-

\footnotetext{
${ }^{26}$ Results elsewhere in mathematical logic, such as the Löwenheim-Skolem theorem and the resolution of Skolem's (merely apparent) paradox, show that mathematical truth, besides being relative and incomplete, is meager in other ways. For example, the first-order theory of real numbers, while complete (in the Gödel sense), still fails to uniquely determine, even up to isomorphism, the model of real numbers that it purports to describe. An upshot of this is that even notions such as countability and infinity are relative (in a particular mathematical sense). It is possible that these results may also have wider philosophical implications, similar to those being argued for here (see, for example, Putnam 1980 and Rashid 2009).
} 
Duhem thesis. As we are forced to recognize that there can be multiple theories of certain sufficiently expressive mathematical systems, so we are forced to recognize that there can be multiple physical theories on a given domain of experience-the underdetermination of theory; or alternatively, as we can modify a sufficiently expressive mathematical theory many different ways by adding any number of different axioms, so too we can modify a physical theory to accommodate any observation-the underdetermination of observation. It is as we said earlier, language cannot completely capture observations (or mathematical ideas). Therefore, by conceding to use language to describe observations (or mathematical ideas), we must allow for flexibility and mutability in its usage. The Quine-Duhem thesis rejects the notion of an absolute truth standard for scientific theories.

3.4.3. Formulating Theories in Lack of a Truth Standard. The decline of truth and the rise of arbitrariness in scientific theories causes one to wonder what are the grounds for selecting correct or even good theories, and second, does such a viewpoint support an anything goes attitude in formulating theories? We start with the second question. We are not, of course, advocating a philosophical position here that there is no world outside the mind, and that we can create any theories we want without regard for what is happening in the outside world. What we are saying is that we cannot know this outside world directly, but only through our interactions with it. Moreover, there is something peculiar about this process of interacting with the outside world that disallows a one-to-one mapping between it and our experiences, thoughts, and languages concerning it, which immediately raises the scepter of incompleteness along with all of its entailments. To be sure, if the outside world was different than what it is now, our theories of it would be different as well. We can only create theories using concepts that are ultimately grounded in our experiences of the outside world (or if perhaps hard-wired genetically at birth). Accordingly, we may say that our theories are conditioned by experience, however incomplete and indeterminable the latter may be. Therefore, while we can create infinitely many correct theories, we cannot create theories irrespective of experience-recall that infinity does not necessarily mean everything.

It may be confusing here as to why we are claiming that theories have to adhere to observations, when we have earlier argued that, by the Quine-Duhem thesis, a theory can accommodate any observation. The subtlety lies in that the Quine-Duhem thesis does not allow theories carte blanche accommodation to observations, but only if the theories undergo appropriate modifications (as explained in Section 2.2.3 and 2.2.5). ${ }^{27}$ Thus any theory, even a seemingly silly one, can be accommodated to observations if the modifications are sufficiently strong. However, if the present amount of perceived incompleteness in the empirical data is small, then all the present theories, including the silly ones, will after modifications converge to a limited number of isomorphic classes as per the UDT requirement theorem (see Section 2.5.1). But because the empirical data can never be complete, it is not possible to determine the final isomorphic class. Thus it could turn out that some theory which has been greatly modified to satisfy the current level of empirical detail may still be a good candidate in a form closer to its original incarnation, given a greater level of empirical detail in the future. For example, closer examination of subatomic phenomena may indicate that space is discrete, as quantum gravity may suggest, which in turn may imply that Lorentz symmetry is violated, returning us to a pre-relativity theoretical framework (Smolin 2003 and references therein). It is in this stronger sense that the QuineDuhem thesis asserts that any theory is compatible with any observation. To sum up, while we cannot just have any arbitrary theory, but must suit it to fit the current level of observational detail, it is always possible, because of the inexhaustible incompleteness of

\footnotetext{
${ }^{27}$ Here we have been talking as if observations are fixed and that it is the theories which must always change. But the same argument can be given from the reverse perspective: we can instead modify the observations by way of modifying the theoretical terms that necessarily impinge on them, given the observational/theoretical distinction failure (as explained in Section 2.2.5). Whether it is theories or observations (or both equally) that are privileged is a matter of perspective in the Quine-Duhem thesis (see Section 2.2.1).
} 
observations, that the original arbitrary theory may better fit the more detailed observations of the future than the currently accepted isomorphic class(es) of theories.

Another point in regards to the second question above is that while we do not advocate an anything goes attitude in formulating scientific theories, the incompleteness of theories, particularly fundamental physical theories, forbids us from asserting that so and so physical phenomena is impossible, e.g., manned flight, teleportation, anti-gravity devices, etc. Incompleteness is not a license for anarchy, but a cause for hope. In this sense, negative results such as incompleteness theorems are not so pessimistic, but leave open the door for new possibilities in the future. Contrariwise, a complete TOE may actually be viewed as a limiting result, in that it says some things are possible but never others.

Now we turn to the first question above: If not only truth, what other standards do we use to judge theories? Aware that the thesis half-named after him left open this question, Quine (1992, 14-15), himself, suggested a pragmatic approach, which is succinctly summarized in his maxim: maximization of simplicity and minimization of mutilation. The basic idea is that among a pool of theories on a given phenomena, we should pick the one that is the most simple, requires the least modification of other theoretical constructs (in a holistic web), and has the broadest scope. An example par excellence of such a theory is, of course, Einstein's general theory of relativity, which unified the laws of gravity and motion into a dramatically simple framework, while achieving a tremendously broad scope in its predictive powers. (It is true that we had to undergo a drastic change in our view of the space-time continuum, but this mutilation is minimal when compared to the plurality of mutilations and complexity of theory we would have to endure in order to retain the Newtonian model.) Moreover, many a physicist (and non-physicist) would readily attach the adjective beautiful to the general theory. Pragmatic and aesthetic attributes, such as simplicity, symmetry, beauty, practicality, predictive powers, scope of the theory, consistency with other theories, among other attributes, do play a role in the formulation and selection of theories. If a physicist was presented with a theory that is experimentally sound, yet enormously complex, not only would she be dissatisfied with it, but she would also wonder if there was not some simpler theory. Truth is not the only bread that scientists eat.

\subsection{Self-Reference in Theories.}

3.5.1. Gödel's Proof. The instrumental use of self-reference in Gödel's proof sheds much light on how self-reference is implicated in scientific theories. Gödel critically recognized that statements about arithmetic (meta-arithmetical statements) can be mirrored within arithmetic. Statements such as " 2 is a factor of 6", "7 is a prime number" and " $2+3=5$ is a theorem of the given arithmetical system" can all be represented as arithmetical formulas. This is possible because (1), as we pointed out in Section 3.3.2, the arithmetic of natural numbers can be used to represent many a formal system with symbols and rules for the manipulation of those symbols, and (2) meta-arithmetical statements are expressible in such a formal system. It should be noted that technically, even without meeting the requirements of the infinity and expressiveness conditions (given in Section 3.3), arithmetical systems can talk about themselves to a certain extent; however, in order to more fully represent themselves and construct the self-referential Gödel formula (to be given below), these requirements must be met.

Having figured out this self-representation capability of arithmetic, Gödel was able to construct an arithmetical formula that when translated meta-arithmetically asserts its own unprovability. Specifically, the formula, say $G$, when translated meta-arithmetically reads, "It is not the case that $G$ is provable within this given arithmetical system." This implies that if the system is consistent, then neither $G$ or its formal negation (not-G) are provable within it. That is to say $G$ cannot be proved or disproved and, hence, is undecidable.

The significance of Gödel's method of proof is that (a) it demonstrates that certain sophisticated formal languages have the capacity for self-reference, and (b) that self-reference in languages leads to undecidable statements. We contend here that any language-cum- 
theory sophisticated enough to describe our experiences of nature also has the capacity for self-reference, and therefore leads to incompleteness in the theory. This is only consistent with our prior contention that any such language-cum-theory also involve, via our sensorialcognitive system, computationally irreducible infinite processes.

A common example used by those explaining Gödel's theorem is the self-referencing capability of a natural language (as opposed to a formal language), like English, as is illustrated in various versions of the Epimenides paradox, or liar paradox: "This statement is false" or "I am lying". A little reflection shows that it is undecidable whether such statements are true or false. Although worth further study, this may be going too far off base for our purposes, as we are interested in languages that are limited to describing natural experience, i.e., languages of scientific theories.

3.5.2. Tarski's Undefinability Theorem and the Theorem of Undefinability of Valid Observations. So far there has been no successful attempt at a formalization of languages for scientific theories. Whether such a formalization is even possible is furthermore not clear (or the even more interesting question of the formalization of any natural language). Because of this we are presently unable to offer a formal incompleteness proof of scientific theories. However, as a temporary measure, we offered in Section 1.7 an informal argument for the undefinability of valid observations. This argument shares some similarities with the proof of Tarski's undefinability theorem (1933).

Applying Gödel's methods of self-reference, Tarski showed that no sufficiently expressive and consistent formal system can provide its own truth definition. Since arithmetic is such a sufficiently expressive formal system, Tarski's theorem applies to it (as well as to many other systems). The counterpart to arithmetical truth in our argument is valid observations. As Tarski showed that a consistent arithmetical theory cannot contain its own truth definition, we have shown that a consistent scientific theory cannot contain its own definition of valid observations. (By "own definition of valid observations", we mean that the definition of observations include those observations that can confirm or disconfirm the given scientific theory.) It is worthwhile to point out the parallels between his theorem and ours.

For his proof Tarski assumed that a given arithmetical theory could contain its own truth definition. From this assumption and using Gödel's methods of self-reference, Tarski was able to generate a contradiction. He constructed an arithmetical formula that when translated meta-arithmetically asserts, not its own unprovability as in Gödel's theorem, but its own falsity. Specifically, the formula, say $T$, when translated meta-arithmetically reads, " $T$ is not a true statement of this given arithmetical system." This statement is an exact analog of the liar paradox; it is true if and only if it is false. This contradiction shows that the truth definition cannot exist within the theory, lest the theory becomes inconsistent. This can also be seen in another way: if the truth definition did exist, then it is incomplete, for $T$ is an arithmetical formula whose truth value cannot be determined from the truth definition.

We can now trace the similarities between Tarski's theorem and ours (please refer to Section 1.7). We assumed that a theory of everything or some other theory could contain its own definition of valid observations, i.e., that it could also serve as its own theory of valid observations. From this assumption and using self reference, we generated a contradiction: the theory can be empirically falsified if and only if it is not false. This contradiction shows that a scientifically falsifiable and consistent theory cannot contain its own definition of valid observations. Moreover, the supposed observation that falsified the hypothetical theory is itself an example of an observation whose validity cannot be determined by the theory.

Both these self-reference arguments show that theories cannot contain their own truth definition or definition of valid observations, as the case may be. They may, however, contain the definitions pertaining to other theories. For example, in the case of arithmetic, its truth definition may be contained in a more expressive theory of arithmetic, and the latter's truth definition may in turn be contained in an even more expressive theory, and so on. Still, in such a scenario, we are merely passing the buck and are led through an endless regress. 
Now, in the case of scientific theories, we can also have some theory define the valid observations that are used to confirm or disconfirm some other theory. Here, as well, we are merely passing the buck and are led through an endless or, perhaps, circular regress (as pointed out in footnote ten in Section 1.7). Moreover, unlike the mathematical case, where we can abstractly construct ever higher mathematical hierarchies, in the scientific case, we can inquire about the theory and validity of all physically possible observations. That is to say, we can inquire about the theory (or the definition) of valid observations, which is in fact what we did in Section 1.7. Since in such a case there is no further theory to turn to, the selfreference argument does not merely show that the theory cannot contain its own definition of valid observations, but that it cannot contain the definition of valid observations.

3.5.3. Self-Reference and the Observational/Theoretical Distinction Failure. The fact that selfreference plays a role in mathematical and scientific incompleteness is telling. When a sophisticated enough theory talks about its world (world of arithmetic, of geometry, of natural phenomena), then because of an inherent self-reference, the theory is also talking about itself. Such theories can be interpreted on two levels: as statements about its world and as meta-statements about itself. This inextricable mixing of reference in theories leads to a natural inseparability between the theory and its world. To put it in stronger terms, selfreference in a theory compromises the separation of the theory and its world, and therefore excludes a one-to-one mapping between the two. In science this inseparability manifests itself as the observational/theoretical distinction failure. We cannot simply put theories on one side and observations on the other; the self-reference inherent in our scientific languages will not allow it. As a more general principle, we may conjecture that self-reference and inseparability exist between thought and experience, between language and thought, between language and experience, and finally, between ourselves and the world outside ourselves. Indeed, we have already postulated in Section 2.1 and 3.2 that scientific incompleteness is connected with a fundamental inseparability in the interaction between nature and our sensorial-cognitive system.

3.6. On the Possibility of an Incompleteness Theorem for Scientific Theories. Our examination has revealed that many of the mechanisms that give rise to and share in mathematical incompleteness could or do also exist in scientific incompleteness. With the exception of complexity, we have been able to identify the common roles played by infinity, arbitrariness, and self-reference in both. Additionally, by employing self-reference, we have been able to construct the equivalent of Tarski's undefinability theorem for scientific observations. These similarities, while helping us to better understand the idea of scientific incompleteness, serve to reinforce the idea and give it a more formal dimension. Furthermore, they seem to suggest that an incompleteness theorem, of sorts, can be shown in science. Here we suggest some possibilities of where we might look for one.

3.6.1. Formalization. We begin with an obvious route: a proof based on the formalization of scientific (or even natural) language. When (or if ever) we achieve such a formalization, we could bring the mathematical methods pioneered by Gödel and others to bear on our attempt. The biggest question here is whether and how such a formalization can be realized. The quantification and mathematization of ever increasing areas of the sciences coupled with efforts to formalize natural language (e.g., Kracht 2003) intimate that the goal of formalization is not unrealistic. We also hinted at this possibility in Sections 2.5.1 and 2.5.2, where we discussed the ideas of science as pattern recognition and data compression. From an information-theoretic/mathematical point of view, some descriptions of physical reality have little information or simple mathematical structure, while others have much information or complex structure. The argument we can put forth here is that scientific disciplines such as biology or, even more so, psychology, having a rich information content, will require considerably more effort to formalize than disciplines such as physics, which has comparably less information. Compelling as the goal of formalization may appear, 
unresolved issues of ontology and semantics cast doubt on whether even the discipline of physics can be given a total formal treatment. Ultimately, further advances in linguistics, mathematics, information theory and other fields are needed before we can consider a proof based on formalization.

3.6.2. Cognitive Science. Since we have been contending that scientific incompleteness is inherent to our sensorial-cognitive interactions with nature, another area where we might find a proof is within the interdisciplinary field of cognitive science. Research into linguistics, neuroscience and artificial intelligence may reveal that experiential and theoretical incompleteness are part of a much broader principle that applies to sentient entities or to living organisms in general. We might find that it is part and parcel of consciousness, a loaded word in itself. Before such an investigation, though, we would need to have a considerably improved understanding of how cognitive processes work, specifically how artificial life forms or sentient entities model and interact within their environments. Clearly, this will require further experimental research.

With this said, an area of cognitive science that presently may offer at least a glimpse of the incompleteness phenomena is the behavioral approach. Maturana and Valera (1980) interpret (scientific) understanding as our attempt, as biological agents, to reach invariance or stability with respect to our environment. Then scientific incompleteness implies that biological agents cannot reach $100 \%$ invariance or stability with respect to their environment. Using the more technical terminology of Maturana and Valera, we can say that an autopoietic system cannot couple perfectly with its environment. For instance, and quoting Bitbol (2001) in his analysis of Piaget (1967), humans use gestures, including words or language, in an attempt to "[carve] out domains of invariance . . . [or] extract elements of stability and iterativity from the Heraclitean flux" for the purpose of reacting predictively to their environment. Then incompleteness implies that such gestures cannot carve out domains of perfect invariance; for example, the meaning of words are not perfectly invariant.

Piaget, if not having come before Maturana and Valera, would say that the ultimate mode of coupling in humans thus far is the culmination of mathematics as a linguistic tool. This has implications for an incompleteness proof based on formalization. For if incompleteness implies that agents cannot achieve a perfect coupling, it would mean, in the case of humans, that our mathematical models cannot completely capture the world. As an interesting possibility, this line of inquiry may benefit in collaboration with the search for a mathematical theory of life, a search first suggested by von Neumann (1966). The behavioral approach seems to offer some hope of a proof and, at the same time, a broader understanding of theoretical incompleteness; though, as is the case with cognitive science in general, considerable more research will be required.

3.6.3. Quantum Physics. As a final suggestion for an area where might find a proof of an incompleteness theorem for scientific theories is within quantum physics, a bedrock of modern science. The field has already produced a limiting result in the form of Heisenberg's Uncertainty Principle. It is not unreasonable to think therefore that further refinement of the theory could yield additional limiting results, particularly an incompleteness theorem. Furthermore, quantum physics possesses many of the key traits found in mathematical and scientific incompleteness. For instance, the uncertainty principle and experiential incompleteness may be related. Also, the uncertainty principle dictates that the outcomes of individual measurements are random or arbitrary; though this form of arbitrariness is not the same kind as in incompleteness, where the arbitrariness manifests itself in deciding the postulates of a theory, there may still be a connection. Similarly, whether there is any connection between the infinities of quantum theory and those of incomplete systems is also unclear. While these parallels may not be convincing enough, the one drawn by self-reference is.

Perhaps the biggest reason to suppose that an incompleteness theorem could be found within quantum physics is because of its self-referential or relational feature. The theory is as 
much a theory of interaction and measurement as it is about subatomic phenomena. It incorporates within its theoretical framework the role played by the observer and her method of interaction with nature. However, it is not just that quantum physics is self-referential that gives us hope, but that it is primarily, if not purely, a theory of interaction that at its core denies the traditional subject-object separation that has been the core presupposition of western thinking for over two millennia.

Though many have gradually come to accept the prominent role quantum theory has given the observer, they continue to retain the traditional view of an external world of objects that stand independent to the observer, i.e., the subject-object separation. In spite of the theory's remarkable predictive powers, the mismatch between this traditional world view and the peculiarities of experimental outcomes has nurtured a continuous philosophical crisis within the theory and spurred many attempts at reconciliation (e.g., the hidden variable theories). It is only in recent times, due to many theoretical and experimental advances (as outlined in Section 1.3.4), that the heavy burden of any potential reconciliation is becoming apparent. These advances make it highly difficult to sustain our most basic notions of object and properties (of objects) in any fundamental theory of subatomic phenomena. Some have taken this outlook as an indication that quantum theory may be a relational theory (Rovelli 1996; Smolin 1995; Bitbol 2001). That is to say it does not purport to describe a world of objects and their corresponding properties, but rather a world of interactions or events relative to an observer. This point is what is relevant for our hopes in finding an incompleteness theorem. The possibility that quantum theory could be a relational theory makes it likely that our future theories will tell us more about the nature of our interaction or relationship with the world, and that somewhere in those theories we may find an incompleteness theorem for science. ${ }^{28}$

As an interesting note, Bitbol believes that the relational character of quantum theory is indicative of a strictly behavioral interpretation of cognitive science, in which cognition is not defined in terms of an agent's ability to represent its environment, but its ability to interact with it. On such a reading, a collaboration between quantum physics and cognitive science may be another route to follow. Additionally, the relational character of quantum theory and the interactive character of behavioral cognitive science seems to suggest that both theories favor the elimination of the observational/theoretical distinction.

The turn in science marked by the advent of quantum theory is not altogether surprising. In science we form theories of our world based on our interactions with the world. Therefore, it is only a matter of time before we are forced to account for the role of our interactions in formulating those theories. It is a reasonable conjecture that any culture (or alien society) will eventually go beyond merely formulating physically descriptive theories and move toward theories which account for the role of their own observer interactions and their processes of ascribing and measuring physical properties. But this is not only the case with science. We can find an analogous situation in mathematics. Just as quantum theory was a reaction to the peculiar discoveries of subatomic phenomena, the meta-theorems (e.g., Gödel's theorem) and other foundational work in mathematics were a reaction to the discovery of paradoxes in set theory. These parallels tell us that, in general, we cannot continue to simply study a subject matter forever, but that foundational crisis will eventually compel us to examine our own methods of inquiry and, in turn, to formulate more relational, holistic theories. In such a landscape, an incompleteness theorem and other meta-theorems begin to seem more natural.

\section{CONCLUSION AND REMARKS}

Implications of the Theory. The philosophical theory outlined here is only a preliminary result. Considerable more research is required in order to gain a clearer understanding of theoretical incompleteness, the novel experience problem, and their many related attributes.

\footnotetext{
${ }^{28}$ Equivalently, information theoretic approaches to quantum theory (e.g., Zeilinger 1999), in which the notion of knowledge is emphasized over the notion of relation, may be fruitful towards this goal.
} 
What these preliminary results tell us is that there is a particular peculiarity about the nature of perception and understanding, about the way sentient entities interact within and understand their environments, and that it is worth examining this peculiarity in more detail.

Second, one should not get the impression that this theory implies that there are absolute truths out there in the universe that we cannot know. Such an implication is indicative of a Platonic mindset, which, as mentioned in Section 3.4, is contrary to the arbitrary nature of theory formulation. This philosophical theory does not draw boundaries on what knowledge is acquirable by humans or other sentient entities; rather, it states that the nature of this knowledge, while it may extend to ever further reaches of our universe, will always be fuzzy around the edges. It may be the case that there is a limit to what we can comprehend-due to the limits of our own brain, for example-but this is not the point being raised here.

With these qualifications out of the way, we can now state what the theory does imply. The two main pillars of the theory, the incompleteness of scientific theories (theoretical incompleteness) and the persistence of the novel experience problem (experiential incompleteness), have already been articulated. Let us then look at some other obvious or not so obvious consequences of the theory. To start with, we should be aware that the incompleteness of scientific theories manifests itself in any format of scientific description. In the definitional (or constituted) approach, incompleteness manifests itself as the inability to give a complete definition due to infinite regress. In the formal (or representative) approach, it manifests itself as the failure to set up a one-to-one correspondence between the symbols of a language and the experienced world. In the axiomatic approach, incompleteness manifests itself as the inability to specify completely the relations between all the primitive terms, i.e., the inability to give a complete axiom list. The reductionistic approach is incomplete on the account of the incompleteness of the definitional and representative approaches. A contextual approach is incomplete due to the inability to specify sufficient context for any scientific term. Finally, a computer model is incomplete due to a limit of observer (or user) interaction, as discussed in Section 2.5.4. Similarly, for other scientific approaches, we can find the role played by incompleteness.

An obvious and fundamental implication, which follows directly from the observational/theoretical distinction failure, is that there is no such thing as pure data in science. This is just restating that there is no such thing as hard observations; nonetheless, this point needs to be reiterated, especially in modern times where scientific authority has achieved a preeminent status. When it is said that so and so data supports some scientific claim, we should not simply take this at face value and assume the claim to be a scientific fact or truth. We should be aware that the corroborative power of the data is contingent upon the broader theoretical context in which it sits. Such a critical mindset allows scientists to seek alternatives to theories that, although "agree with the data", disagree with their own scientific intuition.

The flip side to the above is the implication that there is no such thing as an absolute scientific concept. Just as there are no hard observations, there are no hard concepts either. The meaning of all concepts are incomplete and subject to change. We have seen such changes transpire many times in science, but still we continue to believe that there are at least some absolute concepts. A paragon of such an example is the concept of an object. As we saw in Sections 1.3.4 and 3.6.3, with the advancements of quantum physics, this concept is no longer sacred. Though we may continue to use the term particle to describe subatomic entities, such as electrons and neutrons, the present experimental landscape is making it difficult for us to envision these entities in terms of our traditional conception of localized objects; rather, it steers us toward an abstract quantum formalism, which has yet to receive a consensus interpretation. The concept of an object is a highly convenient theoretical construct based on prior experience. As we improve the resolution of that experience, by observing subatomic phenomena, we see that the concept is changing; we are in need of a new form of data compression. In addition, the concepts of space, time and motion, along with the entire metaphysical framework of causality, are now being re-examined in the light of this new experience with the subatomic. Elsewhere, "fundamental" concepts such as light, mass, 
charge and gravity continue to evolve to this day. There is no Platonic world of scientific concepts. All concepts are founded on experience, and as experiences can change, so can concepts.

Connections with Other Ideas in Philosophy. In their search for truth, philosophers have been a constant witness to a dance between two main notions: rationalism and empiricism (or mind and body, subject and object, analytic and synthetic). Since the beginning of western philosophy, there have been those who advocated purely one or the other notion, but as time went on, there has been a gradual back and forth easing of such a strict dichotomy, culminating with some of the more recent ideas of the twentieth century. The observational/theoretical distinction failure and its many implications are a natural continuation of this recent theme of ideas. They all recognize that rationalism or empiricism as separate schools of philosophy are inadequate to address the central questions of epistemology and ontology. They call for a unification of the above dichotomies into a more holistic theory, and one that takes into account the role of the self.

The observational/theoretical distinction failure itself is comparable to Merleau-Ponty's embodied mind, Heidegger and Gadamer's hermeneutic circle, and Quine's criticism of the analytic/synthetic distinction. Additionally, many of the implications these thinkers have drawn from their respective theses resemble those drawn from the observational/theoretical distinction failure, but in a broader context than that of just scientific theories. Merleau-Ponty ([1945] 1962) argues that because we cannot have disembodied experiences we cannot understand our experiences without ambiguity. Gadamer ([1960] 1975, [1967] 1976) sees understanding as an ongoing process of interaction within the hermeneutic circle of new experiences and prior knowledge, and therefore, like his mentor Heidegger ([1927] 1962, Sections 32-44), rejects that understanding can be objective or complete. Furthermore, Winograd and Flores $(1986,35)$ interpret the hermeneutics of Heidegger and Gadamer to imply that interpretations are not unique, suggestive of the arbitrariness expressed by the Quine-Duhem thesis. The implications of Quine's criticism resulted in his ideas on the philosophy of science, as have already been discussed; however, there is one idea outside the scope of science that is also worth mentioning. This is his thesis of indeterminacy of translation, which states that one can always produce different translation manuals between two natural languages which may be mutually incompatible in some of their translations (Quine 1960, 26-79). Quine's thesis undermines the notion that a given sentence can have a singular meaning.

It is also worth mentioning the work of two other philosophers who have played a role in easing these dichotomies of the west. The latter Wittgenstein, in his advocation of a pragmatic approach to language over a representative one, recognized that for words to be practically useful they must be flexible in meaning. In the exposition of his concept of "language-games", he argues that words can have more than one meaning and share in the meaning of other words-his "family resemblance" (Wittgenstein 1953, aphorisms 2-80). His desire to dull exactness and loosen boundaries in word usage hints at the holistic approach to scientific terminology that was on the horizon. Similarly, Derrida ([1967] 1973, [1967] 1974), in his deconstructive analysis of text, contends that the meaning of texts cannot be explicitly clear, stable or without undecidability. Elsewhere, both Wittgenstein (1953, aphorisms 185243) and Derrida (1994, 37; [1992] 1995, 77) respectively argue that rules do not determine a singular course of action and that decisions require a leap of faith beyond any informative facts. Both these ideas are suggestive of the undecidability of mathematical axioms and the arbitrariness expressed by the Quine-Duhem thesis.

The above philosophers and previously mentioned mathematicians have contributed immensely to philosophy in many different ways; however, a lack of unification among their various ideas has prevented philosophy from seeing more of their implications and feeling their greater impact. The forceful criticism of language by Wittgenstein and Derrida, of the mind/body distinction by Merleau-Ponty, the subject/object separation by Heidegger and Gadamer, and the analytic/synthetic distinction by Quine, all together, raise a compelling 
doubt about our ability, as sentient subjects, to completely or uniquely represent our world. The meta-theorems of Gödel and others have materialized this doubt in the field of mathematics. It is the thesis of this paper that it has also materialized in the field of science. It is therefore most likely the case that no human knowledge is immune to incompleteness. This would then imply that any idea or concept cannot be completely defined, axiomatized or contextualized. It would also mean that a general correspondence theory of truth is unattainable and, moreover, that the notion of truth, itself, is undefinable.

Cause for Optimism: Lessons from Mathematics. The above remarks may convey an immediate negative impression, but if we can view the cup half full, the results obtained here are actually cause for optimism and excitement for not only the scientist, but the non-scientist as well. There are at least three reasons for this. One is that theoretical and experiential incompleteness entails that the scientist work is never done (half empty); this implies that there will always be new and exciting fundamental discoveries to be made (half full). A true explorer would only be saddened if she found out that there were no more worlds left to explore. Second, as was pointed out in Section 3.4.3, theoretical incompleteness allows for novel technological possibilities in the future, permitting the human race to accomplish things that may have been previously thought impossible. Finally, just as the discovery of mathematical incompleteness did not make the mathematician's cause hopeless, but rather opened up whole new worlds of fruitful research (e.g., meta-mathematics), so will the discovery of scientific incompleteness. It suggests for us new questions within the field of linguistics, cognitive science, physics and other areas-foundational questions that bear equally on issues of the physical world as well as the conscious mind. These questions are as exciting as any that has preceded the many productive periods of intellectual discovery in the past. The pursuit of truth, though it may continue forever, will never lead us to a dull road, but to ever more adventurous ones.

\section{ACKNOWLEDGMENTS}

I thank Rocco Lo Bosco for his generous support throughout this project. Without his extensive feedback, editing and our discussions, this paper would not have come to fruition. I also thank Charles Lyons, Richard Mueller and Matt Brown for their many helpful comments, and Jason M. Tyler for the Latin translation of the opening phrase, which may be approximately read as: "All things once dead shall live again."

\section{REFERENCES}

Aczel, Peter (1988). Non-Well-Founded Sets. CSLI Lecture Notes 14, Center for the Study of Languages and Information, Stanford: Stanford University.

Adelberger, E. G., B.R. Heckel, and A.E. Nelson (2003). Tests Of The Gravitational Inverse-Square Law, Annual Review of Nuclear and Particle Science, 53, 77-121.

Adler, Stephen L. (2003). Why Decoherence Has Not Solved the Measurement Problem: A Response to P.W. Anderson, Studies in History and Philosophy of Science Part B, 34, 135-142.

Ahmad, Q.R., et al., SNO Collaboration (2001). Measurement of the Rate of $v_{e}+d \rightarrow p+p+e^{-}$ Interactions Produced by ${ }^{8} \mathrm{~B}$ Solar Neutrinos at the Sudbury Neutrino Observatory, Physical Review Letters 87: 071301.

Amelino-Camelia, Giovanni, et al. (1998). Tests of Quantum Gravity from Observations of $\gamma$-ray Bursts, Nature, 393, 763-765.

Amelino-Camelia, Giovanni (1999). Gravity-Wave Interferometers as Quantum Gravity Detectors, Nature, 398, 216-218.

Anderson, L.H., et al. (2001). Atomic and Condensed Matter Physics in Nuclear Science: Impact, Applications, Interactions. Conference Paper, Nuclear Physics European Collaboration Committee (NUPECC) 2001 Conference, European Science Foundation. [Online Edition]: cited on 1 September 2004, «ttp://www.nupecc.org/iai2001. 
Arkani-Hamed, Nima, Savas Dimopoulos, and Gia Dvali (1998). The Hierarchy Problem and New Dimensions at a Millimeter, Physics Letters B, 429, 263-272.

Aspect, Alain (1981). Experimental Tests of Realistic Local Theories via Bell's Theorem, Physical Review Letters 47, 460-463.

(1982). Experimental Realization of Einstein-Podolsky-Rosen-Bohm Gedankenexperiment. A New Violation of Bell's Inequalities, Physical Review Letters, 49, 91-94.

Babcock, Horace W. (1939). The Rotation of the Andromeda Nebula, Lick Observatory Bulletin, 19(498), 41-51.

Baessler, S., et al. (1999). Improved Tests of the Equivalence Principle for Gravitational Self-Energy, Physical Review Letters, 83, 3585-3588.

von Baeyer, Hans Christian (2003). Information: The New Language of Science. Cambridge, MA: Harvard University Press.

Barrow, John D. (1991). Theories of Everything. Oxford: Clarendon Press.

(1998). Impossibility: The Limits of Science and the Science of Limits. New York: Oxford University Press.

Barut, Asim O. (1995). On the Status of Hidden Variable Theories in Quantum Mechanics, Aperion 2, 97-98.

Bekenstein, Jacob (1974). Generalized Second Law of Thermodynamics in Black-Hole Physics, Physical Review $D$, 9, 3292-3300.

(1981). Universal Upper Bound on the Entropy-to-Energy Ratio for Bounded Systems, Physical Review D, 23, 287-298.

Bell, John S. (1964). On the Einstein-Podolsky-Rosen Paradox, Physics, 1, 195-200.

Bennett, C.L., et al. (2003). First-Year Wilkinson Microwave Anistropy Probe (WMAP) Observations: Preliminary Maps and Basic Results, The Astrophysical Journal Supplement Series, 148, 1-27.

Bennett, G.W., et al., Muon (g-2) Collaboration (2004). Measurement of the Negative Muon Anomalous Magnetic Moment to 0.7 ppm, Physical Review Letters, 92, 161802.

van den Bergh, Sidney (1999). The Early History of Dark Matter, Publications of the Astronomical Society of the Pacific, 111, 657-660.

Bertotti, B., L. Iess, P. Tortora (2003). A Test of General Relativity Using Radio Links with the Cassini Spacecraft, Nature, 425, 374-376.

Bird, Alexander (2003). Kuhn, Nominalism, and Empiricism, Philosophy of Science 70, 690-719.

Bitbol, Michel (2001). Non-Representationalist Theories of Knowledge and Quantum Mechanics, Nordic Journal of Philosophy, 2, 37-61.

Bohm, David (1952). A Suggested Interpretation of Quantum Theory in Terms of 'Hidden Variables', I [and II], Physical Review, 85, 166-193.

Bohm, David, and Jeffrey Bub (1966). A Proposed Solution of the Measurement Problem in Quantum Mechanics by a Hidden Variable Theory, Reviews of Modern Physics, 38, 453-469.

Bohr, Niels (1949). Discussions with Einstein on Epistemological Problems in Atomic Physics, in Paul A. Schilpp (ed.). Albert Einstein: Philosopher-Scientist. Evanston, IL: Library of Living Philosophers.

Breuer, Thomas (1995). The Impossibility of Accurate State Self-Measurements, Philosophy of Science, $62,197-214$

Brown, H.N., et al., Muon (g-2) Collaboration (2000). Improved Measurement of the Positive Muon Anomalous Magnetic Moment, Physical Review D, 62, 091101.

(2001). Precise Measurement of the Positive Muon Anomalous Magnetic Moment, Physical Review Letters, 86, 2227-2231.

Bub, Jeffrey (1995). Fundamental Problems of Quantum Physics, Aperion, 2, 98-100.

Caldwell, Robert (2004). Dark Energy, Physics World May. [Online Edition]: cited on 1 September 2004, http://physicsweb.org/articles/world/17/5/7/1〉.

Calude, C. S., and H. Jürgensen (2005). Is Complexity a Source of Incompleteness? Advances in Applied Mathematics, 35, 1-15.

Campbell, L., et al. (1983). The Sun's Quadrupole Moment and Perihelion Precession of Mercury, Nature, 305, 508-510.

Cappellari, Michele, et al. (1999). Objects in NGC Resolved into Stellar Associations by Hubble Space Telescope Ultraviolet Imaging, The Astropysical Journal, 515, L17-L20.

Cartwright, Nancy (2000). Against the Completability of Science, in M.W.F. Stone and J. Wolff (eds.). The Proper Ambition of Science. London: Routledge, 209-222.

Chaitin, Gregory (1970). On the Difficulty of Computations, IEEE Transactions on Information Theory, 16, 5-9.

(1971). Computational Complexity and Gödel's Incompleteness Theorem, ACM SIGACT News No. $9,11-12$ 
(1974). Information Theoretic Limitations of Formal Systems, Journal of the ACM, 21, 403-424. (1975). Randomness and Mathematical Proof, Scientific American, 232, No. 5 (May), 47-52.

(1990). Algorithmic Information Theory, 3rd Edition. Cambridge: Cambridge University Press.

[Online Edition]: cited on 20 November 2004, 〈http:/ /www.umcs.maine.edu/ chaitin/cup.pdf) (2000). A Century of Controversy over the Foundations of Mathematics, in C.S. Calude and G. Paun (eds.). Finite Versus Infinite. London: Springer-Verlag, 75-100.

Choi, S.-K., et al., Belle Collaboration (2003). Observation of a narrow charmoniumlike state in exclusive B+/--->K+/-pi+pi-J/psi decays. Physical Review Letters, 91, 262001.

Church, Alonzo (1936). An Unsolvable Problem of Elementary Number Theory, American Journal of Mathematics 58, 345-363.

Churchland, Paul (1979). Scientific Realism and the Plasticity of Mind. Cambridge: Cambridge University Press.

- (1988). Perceptual Plasticity and Theoretical Neutrality: A Reply to Jerry Fodor, Philosophy of Science, 55, 167-187.

Cimatti, A., et al. (2004). Old Galaxies in the Young Universe, Nature, 430, 184-187.

Cohen, Paul J. $(1963,1964)$. The Independence of the Continuum Hypothesis [and Part II], Proceedings of the National Academy of Sciences of the U.S.A., 50, 1143-1148; 51, 105-110.

Contaldi, Carlo R., et al. (2003). Suppressing the Lower Multipoles in the CMB Anisotropies, Journal of Cosmology and Astroparticle Physics, 7, 002.

Davier, M., S. Eidelman, A. Hocker, and Z. Zhang (2003). Updated Estimate of the Muon Magnetic Moment Using Revised Results from $e^{+} e^{-}$Annihilation, The European Physical Journal C, 31, 503-510. Davies, Paul (1992). The Mind of God. New York: Touchstone.

Deffayet, Cedric, Gia Dvali, and Gregory Gabadadze (2002). Accelerated Universe from Gravity Leaking to Extra Dimensions, Physical Review D, 65, 044023.

Derrida, Jacques ([1967] 1974). Of Grammatology. Translated by Gayatri Spivak. Baltimore: The John Hopkins University Press.

([1967] 1973). Speech and Phenomena, and Other Essays on Husserl's Theory of Signs. Translated by David B. Allison. Evanston, IL: Northwestern University Press.

- ([1992] 1995). The Gift of Death. Translated by David Wills. Originally published as Donner la mort in L'éthique du don, Jacques Derrida et la pensée du don (Paris: Métailié-Transition). Chicago: University of Chicago Press.

(1994). Nietzsche and the Machine: Interview with Jacques Derrida (Interviewed by Richard Beardsworth). Journal of Nietzsche Studies, Issue 7.

Dieks, D. (1991). On Some Alleged Difficulties on the Interpretation of Quantum Mechanics, Synthese, 86, 77-86.

Duhem, Pierre ([1906] 1954). The Aim and Structure of Physical Theory. Reprint. Translated by Philip P. Wiener. Originally published as La théorie physique: son objet, et sa structure (Paris: Marcel Rivière \& Cie). Princeton: Princeton University Press.

Durrani, Matin, and Peter Rodgers (1999). Physics: Past, Present, Future, Physics World December. [Online Edition]: cited on 15 January 2004, 〈http:/ / physicsweb.org/articles/world/12/12/14/1〉.

van Dyck, Robert S. Jr., Paul B. Schwinberg, and Hans G. Dehmelt (1987). New High-Precision Comparison of Electron and Positron $g$ Factors, Physical Review Letters, 59, 26-29.

Efstathiou, G. (2003). Is the Low Cosmic Microwave Background Quadrupole a Signature of Spatial Curvature, Monthly Notices of the Royal Astronomical Society, 343, L95-L98.

Einstein, Albert, and Leopold Infeld ([1938] 1966). The Evolution of Physics, New Edition. New York: Simon \& Schuster.

Evans, Denis J., E.G.D. Cohen, and G.P. Morriss (1993). Probability of Second Law Violations in Shearing Steady States, Physical Review Letters, 71, 2401-2404.

Evdokimov, A.V., et al., SELEX Collaboration (2004). First Observation of a Narrow Charm-Strange Meson DsJ(2632) $\rightarrow$ Ds eta and DO $K^{+}$. Fermilab-Pub-04/087-E, Submitted to Physical Review Letters. [Online Edition]: cited on 15 October 2004, 〈http:/ /www.arxiv.org/abs/hep-ex/0406045».

Everett, H., III (1957). 'Relative State' Formulation of Quantum Mechanics, Reviews of Modern Physics $29,454-462$.

Feynman, Richard (1967). The Character of Physical Law. Cambridge, MA: MIT Press. (1985). QED: The Strange Theory of Light and Matter. Princeton: Princeton University Press.

Fodor, Jerry (1984). Observation Reconsidered, Philosophy of Science, 51, 23-43. (1988). A Reply to Churchland's 'Perceptual Plasticity and Theoretical Neutrality', Philosophy of Science, 55, 188-198.

Forti, Marco, and Furio Honsell (1983). Set Theory with Free Construction Principles, Annali Scuola normale superior, 10, 493-522. 
van Fraassen, B.C. (1989). The Charybdis of Realism: Epistemological Implications of Bell's Inequality, in J. Cushing and E. McMullin (eds.). Philosophical Consequences of Quantum Theory. Notre Dame: University of Notre Dame Press.

(1991). Quantum Mechanics: An Empiricist View. Oxford: Clarendon Press.

Friedman, Jonathan R., et al. (2000). Quantum Superposition of Distinct Macroscopic States, Nature. $406,43-46$.

Gadamer, Hans-Georg ([1960] 1975). Truth and Method. Translated and edited by Garrett Barden and John Cumming. New York: Seabury Press.

_[1967] 1976). Philosophical Hermeneutics. Translated and edited by David E. Linge. Berkeley, University of California Press.

Ghirardi, G.C., A. Rimini, \& T. Weber (1986). Unified Dynamics for Microscopic and Macroscopic Systems, Physical Review D34, 470-491.

Gisin, N., \& I.C. Percival (1992). The Quantum-State Diffusion Model Applied to Open Systems, Journal of Physics A, 25, 5677-5691.

Glazebrook, Karl, et al. (2004). A High Abundance of Massive Galaxies 3-6 Billion Years after the Big Bang, Nature, 430, 181-184.

Gleason, A. M. (1957). Measures on the Closed Subspaces of a Hilbert Space, Journal of Mathematics and Mechanics, 6, 885-893.

Gödel, Kurt (1931). Über Formal Unentscheidbare Sätze der Principia Mathematica und Verwandter Systeme, I, Monatshefte für Mathematik und Physik 38, 173-198. English translation in van Heijenoort (1976).

(1940). Consistency of the Continuum Hypothesis. Princeton: Princeton University Press.

Greene, Brian (2003). The Elegant Universe. New York: Vintage Books.

Hall, Asaph (1894). A Suggestion in the Theory of Mercury, Astronomical Journal, 14, 49-51.

Hawking, Stephen (1988). A Brief History of Time. New York: Bantam Books.

(2002). Gödel and the End of Physics. Lecture, Dirac Centennial Celebration, Cambridge: University of Cambridge.

(2003). Cosmology from the Top Down. Proceedings of the Davis Cosmic Inflation Meeting, Davis: University of California. [Online Edition]: cited on 1 February 2004,

〈http://www.arxiv.org/abs/astro-ph/0305562〉.

Heavens, Alan, et al. (2004). The Star-formation History of the Universe from the Stellar Populations of Nearby Galaxies, Nature 428, 625-627.

Heidegger, Martin ([1927] 1962). Being and Time. Translated by John Macquarrie and Edward Robinson. New York: Harper \& Row.

van Heijenoort, Jean (ed.) (1976). From Frege to Gödel: A Source Book in Mathematical Logic, 18791931, 3rd ed. Cambridge, Mass: Harvard University Press.

Hendry, John (1980). Newton's Theory of Colour, Centaurus 23, 230-251.

Hoefer, Carl and Alexander Rosenberg (1994). Empirical Equivalence, Underdetermination, and Systems of the World, Philosophy of Science, 61, 592-607.

Hofstadter, Douglas (1979). Gödel, Escher, Bach: An Eternal Golden Braid. New York: Basic Books.

Hollik, Wolfgang (2002). Electroweak Precision Analysis. Conference Paper, 10th International Conference on Supersymmetry and Unification of Fundamental Interactions, Hamburg. [Online Edition]: cited on 15 October 2004,

〈http://www-library.desy.de/preparch/desy/proc/proc02-02/Proceedings/pl.2/hollik_pr.pdf〉.

Horgan, John (1996). The End of Science. Reading, Mass: Addison-Wesley.

Hoyle, C.D., et al. (2001). Submillimeter Test of the Gravitational Inverse-Square Law: A Search for 'Large' Extra Dimensions, Physical Review Letters, 86, 1418-1421.

Hughes, R.I.G. (1989). The Structure and Interpretation of Quantum Mechanics. Cambridge, MA: Harvard University Press.

Ilic, B., et al. (2004). Attogram Detection Using Nanoelectromechanical Oscillators, Journal of Applied Physics, 95, 3694-3703.

Jaki, Stanley (1966). The Relevance of Physics. Chicago: University of Chicago Press.

Knecht, Marc, and Andreas Nyffeler (2002). Hadronic Light-by-Light Corrections to the Muon $g$-2: The Pion-Pole Contribution, Physical Review D, 65, 073034.

Knecht, Marc (2003). The Anomalous Magnetic Moment of the Muon: A Theoretical Introduction. CPT2003/P.4525. [Online Edition]: cited on 15 October 2004, 〈http://www.arxiv.org/abs/hep$\mathrm{ph} / 0307239$.

Kochen, S., \& E.P. Specker (1967). The Problem of Hidden Variables in Quantum Mechanics, Journal of Mathematics and Mechanics 17, 59-87.

Kracht, Marcus (2003). The Mathematics of Language. Berlin: Mouton de Gruyter. 
Kuhn, Thomas (1970). The Structure of Scientific Revolutions, 2nd Edition. Chicago: University of Chicago Press.

Laudan, L., \& J. Leplin (1991). Empirical Equivalence and Underdetermination, Journal of Philosophy $88,449-472$.

LeBach, D.E., et al. (1995). Measurement of the Solar Gravitational Deflection of Radio Waves Using Very-Long Baseline Interferometry, Physical Review Letters, 75, 1439-1442.

Leggett, A.J. (2002). Testing the Limits of Quantum Mechanics: Motivation, State of Play, Prospects, Journal of Physics: Condensed Matter 14, R415-R451.

Linde, Andrei (1994). The Self-Reproducing Inflationary Universe, Scientific American, 271, No. 5 (November), 48-55.

Long, J.C., H.W. Chan, \& J.C. Price (1999). Experimental Status of Gravitational-Strength Forces in the Sub-Centimeter Regime, Nuclear Physics B539, 23-34.

Lindley, David (1993). The End of Physics: The Myth of a Unified Theory. New York: Basic Books.

Luminet, Jean-Pierre, et al. (2003). Dodecahedral Space Topology as an Explanation for Weak WideAngle Temperature Correlations in the Cosmic Microwave Background, Nature, 425, 593-595.

Marciano, W.J. (2003). Precision Measurements and New Physics, Journal of Physics G, 29, 225-234.

Matiyasevich, Yuri (1970). Enumerable sets are Diophantine (Russian). Doklady Akademii Nauk SSSR 191 (2), 279-282. English translation in Soviet Mathematics, 11 (2), 354-358.

Maturana, Humberto R., and Francisco Varela (1980). Autopoiesis and Cognition: The Realization of the Living. Dordrecht: Reidel.

McAllister, James W. (1997). Phenomena and Patterns in Data Sets, Erkenntnis 47, 217-228.

(2003). Effective Complexity as a Measure of Information Content, Philosophy of Science, 70, 302-307.

McGuire, John E., and Martin Tamny (1983). Certain Philosophical Questions: Newton's Trinity Notebook. Cambridge: Cambridge University Press.

Merleau-Ponty, Maurice ([1945] 1962). Phenomenology of Perception. Translated by Colin Smith. London: Routledge and Kegan Paul.

Mermin, David (1998). What is Quantum Mechanics Trying to Tell Us?, American Journal of Physics, $66,753-767$.

Mester, J., et al. (2001). The STEP Mission: Principles and Baseline Design, Classical and Quantum Gravity, 18, 2475-2486.

Miller, J.P., Muon (g-2) Collaboration (2002). The Muon Anomaly: Experiment and Theory, International Journal of Modern Physics A, 17, 3318-3335.

Milgrom, Mordehai (1983a). A Modification of the Newtonian Dynamics as a Possible Alternative to the Hidden Mass Hypothesis, The Astrophysical Journal, 270, 365-370.

Milgrom, Mordehai, and Robert H. Sanders (2003). Modified Newtonian Dynamics and the 'Dearth of Dark Matter in Ordinary Elliptical Galaxies, The Astrophysical Journal, 599, L25-L28.

Moffat, J.W. (2004). Modified Gravitational Theory as an Alternative to Dark Energy and Dark Matter. [Online Article]: cited on 15 October 2004, http:/ /www.arxiv.org/abs/astro-ph/0403266).

Moore, Cristopher (1990). Unpredictability and Undecidability in Dynamical Systems, Physical Review Letters 64, 2354-2357.

Murawski, Roman (1998). Undefinability of Truth. The Problem of Priority: Tarski vs. Gödel, History and Philosophy of Logic, 19, 153-60.

Nesvizhevsky, Valery, et al. (2002). Quantum States of Neutrons in the Earth's Gravitational Field, Nature, 415, 297-299.

von Neumann, John ([1932] 1955). Mathematical Foundations of Quantum Mechanics. Translated by R.T. Beyer. Princeton: Princeton University Press.

(1966). Theory of Self-Reproducing Automata. Urbana, IL: University of Illinois Press.

Newton, Isaac ([1730] 1952). Optiks, 4th Edition. New York: Dover.

Paredes, Belén, et al. (2004). Tonks-Girardeau Gas of Ultracold Atoms in an Optical Lattice, Nature, 429, 277-281.

Perlmutter, S., et al. (1998). Discovery of Supernova at Half the Age of the Universe, Nature 391, 51-54.

Piaget, Jean (1967). Biologie et Connaissance. Paris: Gallimard.

Plazanet, M. et al. (2004). Freezing on Heating of Liquid Solutions, The Journal of Chemical Physics, 121, 5031-5034.

Popper, Karl (1959). The Logic of Scientific Discovery. New York: Basic Books.

Presburger, Mojzesz ([1929] 1991). On the Completeness of a Certain System of Arithmetic of Whole Numbers in Which Addition Occurs as the Only Operation, Translated by Dale Jacquette, History and Philosophy of Logic, 12, 225-233. Originally published as Über die Vollständigkeit eines gewissen 
Systems der Arithmetik ganzer Zahlen, in welchem die Addition als einzige Operation hervortritt, Sparawozdanie z I Kongresu matematyków krajów slowianskich, Warszawa, 92-101.

Putnam, Hilary (1980). Models and Reality, The Journal of Symbolic Logic 45, 464-482.

Quine, W.V.O. (1951). Two Dogmas of Empiricism, The Philosophical Review, 60, 20-43. (1960). Word and Object. Cambride, MA: MIT Press.

(1992). Pursuit of Truth, Revised Edition. Cambridge, MA: Harvard University Press.

Rashid, Salim (2009). Underdetermination, Multiplicity, and Mathematical Logic. [Online Article]: cited on 3 May 2011, ‘ttp://philsci-archive.pitt.edu/4905/〉.

Rovelli, Carlo (1996). Relational Quantum Mechanics, International Journal of Theoretical Physics, 35, 1637-1678.

Rowe, M.A., et al. (2001). Experimental Violation of Bell's Inequality with Efficient Detection, Nature, 409, 791-794.

Rubin, Vera C., and W. Kent Ford, Jr. (1970). Rotation of the Andromdea Nebula from a Spectroscopic Survey of Emission Regions, The Astrophysical Journal, 159,379-404.

Ruphy, Stéphanie (2003). Is the World Really 'Dappled'? A Response to Cartwright's Charge against 'Cross-Wise Reduction', Philosophy of Science, 70, 57-67.

Sankey, Howard (1997). Incommensurability: The Current State of Play, Theoria, 12, 425-445.

Skolem, Thoralf (1930). Über einige Satzfunktionem in der Arithmetik, Skrifter Utgitt av Det Norske Videnskaps-Akademi i Oslo, I. Mat.-Naturv.

Smith, Sinclair (1936). The Mass of the Virgo Cluster, The Astrophysical Journal 83, 23-30.

Smolin, Lee (1995). The Beckenstein Bound, Topological Quantum Field Theory and Pluralistic Quantum Field Theory. [Online Article]: cited on 20 November 2004, 〈http://arxiv.org/abs/grqc/9508064).

(1997). The Life of the Cosmos. New York: Oxford University Press.

(2003). How Far Are We from the Quantum Theory of Gravity? [Online Article]: cited on 10 November 2004, http:/ / arxiv.org/abs/hep-th/0303185.

Solomonoff, Ray (1964). A Formal Theory of Inductive Inference, Part 1 [\&2], Information and Control 7, 1-22 \& 224-254.

Sommerer, John C., \& Edward Ott (1996). Intermingled Basins of Attraction: Uncomputability in a Simple Physical System, Physics Letters A 214, 243-251.

Soussa, M.E., \& R. P. Woodard (2003). A Nonlocal Metric Formulation of MOND, Classical and Quantum Gravity, 20, 2737-2751.

Suppes, Patrick (1962). Models of Data, in E. Nagel, P. Suppes, and A. Tarski (eds.). Logic, Methodology, and Philosophy of Science. Stanford: Stanford University Press, 252-261.

(1978). The Plurality of Science, in P.D. Asquith and I. Hacking (eds.). PSA 1978, Volume 2. Philosophy of Science Association, 3-16.

Susskind, Leonard (2003). The Anthropic Landscape of String Theory. [Online Article]: cited on 10 November 2004, ‘http:/ /arxiv.org/abs/hep-th/0302219〉.

Tarski, Alfred (1933). The Concept of Truth in the Languages of the Deductive Sciences (Polish). Prace Towarzystwa Naukowego Warszawskiego, Wydzial III Nauk Matematyczno-Fizycznych 34. English translation in Tarski (1983).

(1983). Logics, Semantics, Metamathematics, 2nd ed. Translated by J.H. Woodger and edited by J. Corcoran. Indianapolis: Hackett Publishing Company.

Touboul, Pierre, and Manuel Rodrigues (2001). The MICROSCOPE Space Mission, Classical and Quantum Gravity, 18, 2487-2498.

Traub, Joseph F. (1996). On Reality and Models, in J. Casti and A. Karlqvist (eds.). Boundaries and Barriers: On the Limits to Scientific Knowledge. Reading, MA: Addison-Wesley. 29-31. (1997a). Do Negative Results from Formal Systems Limit Scientific Knowledge? Complexity, 3,

(1997b). Non-Computability and Intractability: Does it Matter to Physics? Technical Report, Columbia University Department of Computer Science.

Turing, Alan (1936). On Computable Numbers, with an Application to the Entscheidungsproblem, Proceedings of the London Mathematical Society, 42, 230-265.

Turneaure, J.P., et al. (2003). Development of the Gravity Probe B Flight Mission, Advances in Space Research, 32, 1387-1396.

van der Wal, Caspar H., et al. (2000). Quantum Superposition of Macroscopic Persistent-Current States, Science, 290, 773-777.

Wang, G.M., et al. (2002). Experimental Demonstration of Violations of the Second Law of Thermodynamics for Small Systems and Short Time Scales, Physical Review Letters, 89, 050601.

Weinberg, Steven (1992). Dreams of a Final Theory. New York: Pantheon. 
Weinstein, Steven (2003). Objectivity, Information, and Maxwell's Demon, Philosophy of Science, 70, 1245-1255

Weiss, Peter (2003). A Spin through Space-Time, Science, 164, 280.

Wigner, E.P. (1961). Remarks on the Mind-Body Question, in I.J. Good (ed.). The Scientist Speculates. London: Heinemann.

Wiles, Andrew (1995). Modular Elliptic Curves and Fermat's Last Theorem, Annals of Mathematics 142, 443-551.

Will, Clifford M. (2001). The Confrontation between General Relativity and Experiment, Living Reviews in Relativity 4, 4. [Online Article]: cited on 1 October 2004, ‘http:/ /www. livingreviews.org/lrr-20014>.

Williams, J. G., et. al. (2002). Lunar Laser Tests of Gravitational Physics, in V. G. Gurzadyan, R. T. Jantzen, \& R. Ruffini (eds.). Proceedings of The Ninth Marcel Grossmann Meeting. Singapore: World Scientific, 1797-1798.

Winograd, Terry, and Fernando Flores (1986). Understanding Computers and Cognition. Norwood, NJ: Ablex Publishing Corporation.

de Witt, B.S. (1970). Quantum Mechanics and Reality, Physics Today 23, 30-55.

Wittgenstein, Ludwig (1953). Philosophical Investigations. Translated by G.E.M Anscombe and edited by G.E.M Anscombe and R. Rhees. Oxford: Blackwell.

Wolfram, Stephen (1985). Undecidability and Intractability in Theoretical Physics, Physical Review Letters, 54, 735-738. (2002). A New Kind of Science. Champaign: Wolfram Media.

Zeh, H.D. (2003). Basic Concepts and their Interpretation, in E. Joos (ed.). Decoherence and the Appearance of a Classical World in Quantum Theory, 2nd Edition. Heidelberg: Springer-Verlag. [Online Edition]: cited on 10 November 2004, 〈http:/ / arxiv.org/abs/quant-ph/9506020〉.

Zehavi, Idit, and Avishai Dekel (1999). Evidence for a Positive Cosmological Constant from Flows of Galaxies and Distant Supernovae, Nature, 401, 252-254.

Zeilinger, Anton (1998). Fundamentals of Quantum Information, Physics World March. [Online Edition]: cited on 10 November 2004, 〈http:/ / physicsweb.org/articles/world/11/3/9/1〉. (1999). A Foundational Principle for Quantum Mechanics, Foundations of Physics, 29, 631-643.

Zwicky, Fritz (1933). The Red Shift of Extragalactic Nebulas, Helvetica Physica Acta, 6, 110-127. 